Article

\title{
Exploring Changes in Land Surface Temperature Possibly Associated with Earthquake: Case of the April 2015 Nepal Mw 7.9 Earthquake
}

\author{
Shunyun Chen ${ }^{1, *} \mathbb{C}$, Peixun Liu ${ }^{1}$, Tao Feng ${ }^{2}$, Dong Wang ${ }^{2}$, Zhonghu Jiao ${ }^{1}\left[\right.$, Lichun Chen ${ }^{1,3}$, \\ Zhengxuan $\mathrm{Xu}^{2}$ and Guangze Zhang ${ }^{2}$ \\ 1 State Key Laboratory of Earthquake Dynamics, Institute of Geology, Beijing 100029, China; \\ liupx@ies.ac.cn (P.L.); jzh@ies.ac.cn (Z.J.); dzsclc@ies.ac.cn (L.C.) \\ 2 China Railway Eryuan Engineering Group Co. Ltd., Chengdu 610031, China; fengtao@ey.crec.cn (T.F.); \\ wangdong13@ey.crec.cn (D.W.); xuzhengx@ey.crec.cn (Z.X.); zhanggz@ey.crec.cn (G.Z.) \\ 3 College of Earth Sciences, Guilin University of Technology, Guilin 541006, China \\ * Correspondence: chenshy@ies.ac.cn; Tel.: +86-106-200-9112
}

Received: 20 February 2020; Accepted: 23 March 2020; Published: 26 March 2020

\begin{abstract}
Satellite thermal infrared remote sensing has received worldwide attention in the exploration for earthquake precursors; however, this method faces great controversy. Obtaining repeatable phenomena related to earthquakes is helpful to reduce this controversy. In this paper, a total of 15 or 17 years of Moderate-resolution Imaging Spectroradiometer (MODIS)/Aqua and MODIS/Terra satellite remote sensing land surface temperature (LST) products is selected to analyze the temperature changes before and after the Mw 7.9 earthquake in Nepal on 25 April 2015 and to explore possible thermal information associated with this earthquake. Major findings are given as follows: (1) from the time course, the temperature slowly cooled before the earthquake, reached a minimum at the time of the earthquake, and returned to normal after the earthquake. Since these changes were initiated before the earthquake, they may even have been precursors to the Nepal earthquake. (2) From the space distribution, the cooling areas correspond to the seismogenic structure during the earthquake. These cooling areas are distributed along the Himalayas and are approximately $1300 \mathrm{~km}$ long. The widths of the East and West sides are slightly different, with an average temperature decrease of $5.6^{\circ} \mathrm{C}$. For these cooling areas, the Western section is approximately $90 \mathrm{~km}$ wide and $500 \mathrm{~km}$ long; the East side is approximately $190 \mathrm{~km}$ wide and $800 \mathrm{~km}$ long. The Western side of the cooling strips appeared before the earthquake. In short, these kinds of spatial and temporal changes are tectonically related to the earthquake and may have been caused by the tectonic activity associated with the Nepal earthquake. This process began before the earthquake and therefore might even be potentially premonitory information associated with the Nepal earthquake.
\end{abstract}

Keywords: Nepal Mw 7.9 earthquake; land surface temperature; the temperature change associated with earthquake; MODIS/Aqua; MODIS/Terra

\section{Introduction}

For a period of time in the past, it had been strongly claimed by some researchers that earthquakes are unpredictable catastrophes, based on the pervasive hypothesis that the Earth's crust is in a state of perpetual self-organized criticality in which any small earthquake may cascade into a large event $[1,2]$. The view of earthquake unpredictability seemed to have swept the world. However, it has been substantiated by observational evidence that this hypothesis is far from the truth, at least in regard to large earthquakes [3,4]. Even a self-organized system like weather forecasting can be well predicted to a certain extent. Because great earthquakes usually cause serious damage to humanity and society, the 
predictability of damaging earthquakes is widely paid attention to, and actually, a lot of attempts over the world have been made to investigate precursors of deadly earthquakes.

Among many methods for exploring the premonitory signals before an earthquake, satellite thermal infrared remote sensing has received worldwide attention in the exploration of earthquake precursors [5-7]. Many examples of thermal infrared anomalies have been reported [6,8-15]. A variety of anomaly extraction methods and their possible physical mechanisms have also been proposed [9,11,16-24]. However, there is still a high degree of controversy regarding satellite thermal infrared anomalies $[25,26]$. This controversy is, at least partially, is related to the complexity in anomalies of thermal infrared remote sensing. The thermal infrared information received by satellites is mainly thermal radiation from the land surface, which has been modified by the atmosphere, including a large variety of complex meteorological and other non-seismic factors. Eliminating the influence of these factors is very important for understanding these kinds of thermal anomalies [27]. Temperature changes produced by meteorological factors can reach the order of a few or even a dozen degrees Celsius, and the abnormal information related to earthquakes may be completely submerged by meteorological changes. Having appropriate methods and detailed data processing to extract thermal information before an earthquake is necessary. In this paper, taking the April $2015 \mathrm{Nepal} \mathrm{Mw} \mathrm{7.9}$ Earthquake as a case, the following aspects are considered. (1) First, determine the known information as thoroughly as possible, especially non-tectonic factors such as atmospheric and solar influences, and then look for possible earthquake-related information from the remaining information. (2) If a piece of information is related to an earthquake, it should be spatially related to the seismogenic structure; it should temporally include coseismic information; and even if there is an earthquake precursor, the change should become more significant at the occurrence of the earthquake. (3) The earthquake-related phenomenon should be repeated when using data from different satellites. The processing of satellite data can also generate uncertainty, especially for long-term satellite data. Using different satellite data for verification is best. If different satellites detect the same phenomenon, then at least the phenomenon itself is reliable.

A strong earthquake shook Nepal on 25 April 2015, with a moment magnitude of Mw 7.9 and an initial rupture depth of approximately $12-15 \mathrm{~km}$ [28]. The epicenter of the mainshock was at $84.708^{\circ} \mathrm{E}$, $28.147^{\circ} \mathrm{N}$ and occurred in Gorkha district of Nepal [29]. The earthquake caused severe damage to life and property and was perhaps the worst natural hazard to strike Nepal since the 1934 quake [30]. The Mw 7.9 earthquake in Nepal occurred on the Qinghai-Tibet Plateau, where the mountains are steep, the terrain is complex, and in situ field observations face many difficulties. Satellite remote sensing can provide information on the evolution of changes in time and space. Discovering some earthquake-related clues is possible, which would be valuable for increasing the understanding of the Nepal earthquake [25,30,31] [26]. In this paper, following the above-mentioned three points, a total of 15 or 17 years of both MODIS/Aqua and MODIS/Terra satellite remote sensing LST products from 2002 or 2000 to 2016 are selected to investigate the temperature changes before and after the $\mathrm{Mw}$ 7.9 earthquake in Nepal on 25 April 2015 and to explore the thermal anomalies that may have been associated with the earthquake.

\section{The Geological Background and Research Areas}

\subsection{Geological Background of Southern Margin of the Qinghai-Tibet Plateau}

Since the Eocene, the Indian Plate and the Eurasian Plate have collided along a plate boundary of approximately $2500 \mathrm{~km}$ to form the Himalayan orogenic belt. The cumulative crustal shortening is approximately 2000-3000 km [32]. The collision between the plates produced some thrust faults, like the main boundary thrust (MBT), parallel to the Himalayan arc on the Southern side of the Himalayas (Figure 1a). The main boundary thrust, which constitutes the boundary between the Low Himalaya and the sub-Himalaya, consists of a series of thrust faults with Northern dips. In some locations, the MBT causes the pre-Tertiary strata to thrust. Above the Quaternary strata [33], the Southernmost 
fault is the main frontier thrust (MFT), which constitutes the boundary between the Indian Plate and the Qinghai-Tibet block and can be seen on the surface, causing the Cirqueke Formation of the New World to be thrust onto the Quaternary sediments of the Ganges Plain [34]. These nearly parallel, North-dipping thrust faults on the surface merge downward into a unified detachment fault, the main Himalayan thrust (MHT) [35]. Many large earthquakes occurred in these thrust faults in the Southern margin of the Qinghai-Tibet Plateau [29].

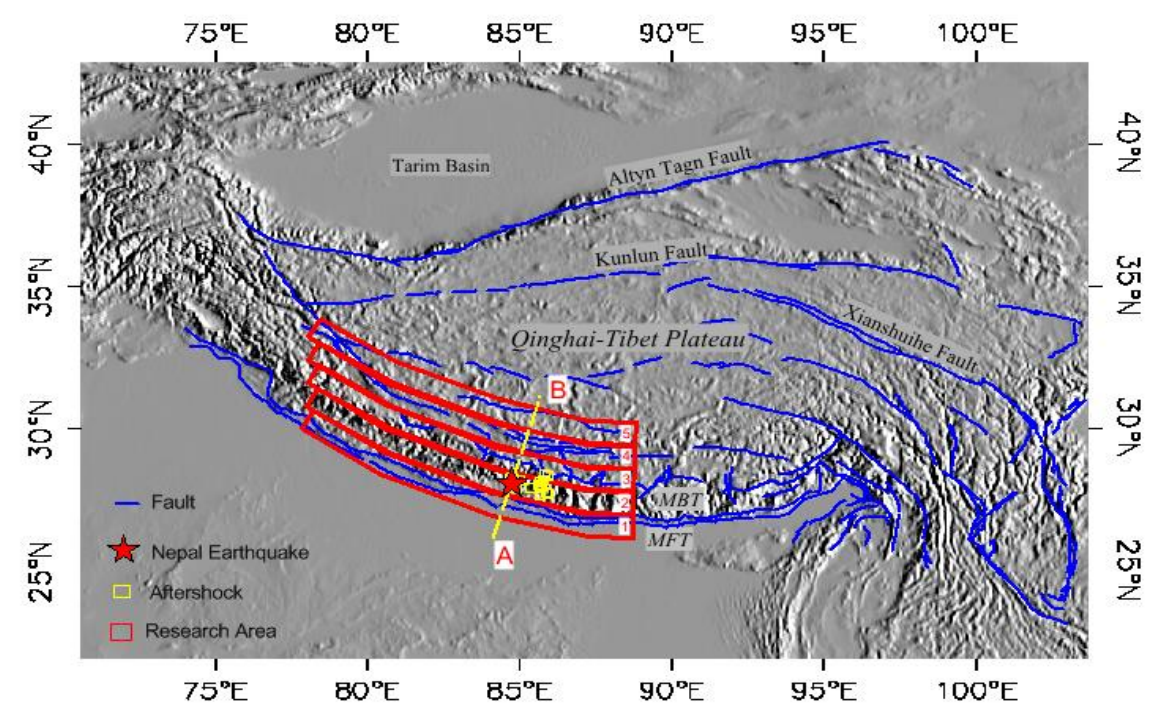

(a)

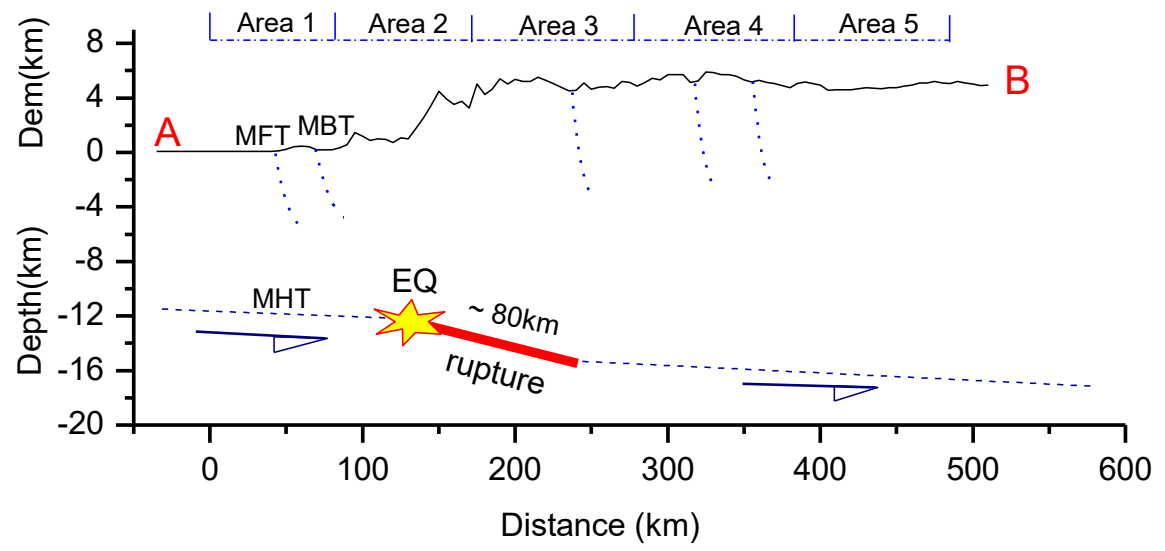

(b)

Figure 1. Schematic map of the fault system of the Qinghai-Tibet Plateau and diagrams of the research areas. (a) Spatial partition, where numbers 1,2,.., 5 represent different areas, and AB is the profile position. (b) The topographic profile along $\mathrm{AB}$ and research areas (Area 1, Area 2,..., Area 5); the seismic rupture is obtained from Reference [36].

On 25 April 2015, the Mw 7.9 earthquake in Nepal occurred in the Southern foothills of the Himalayas. The epicenter of the mainshock was at $84.708^{\circ} \mathrm{E}, 28.147^{\circ} \mathrm{N}$, and the initial rupture depth was approximately $12-15 \mathrm{~km}$ [28]. The aftershocks $(M w \geq 5)$ that occurred on 25 April 2015 were given in Figure 1a. The focal mechanism solution indicates that the earthquake was a low-angle thrust-type earthquake [29]. Field investigations have not found that the Mw 7.9 earthquake in Nepal formed a surface rupture zone on the main fault in the Southern foothills of the Himalayas. The tectonic nature of the Southern foothills of the Himalayas is a thin-skin structure, which is characterized by the steepening slope of the shallow fault (approximately $7^{\circ}$ ) and the deep fault (approximately $11^{\circ}$ ). 
According to the seismic wave inversion results [36], the coseismic rupture extends $\sim 80 \mathrm{~km}$ toward the North (Figure $1 \mathrm{~b}$ ) and $\sim 100 \mathrm{~km}$ toward the Southeast. Regarding the coseismic stress drop for the 2015 Nepal Mw 7.9 earthquake, the values given by different scholars are quite different. Some researchers have reported that the stress drops were approximately 3.0-3.2 MPa [37]; however, other researchers assume a circular crack model and their spectral characteristics to estimate stress drops of $22.7 \pm 1.9 \mathrm{MPa}$ for the mainshock [38]. These values for the stress drops are highly dependent on the model [37].

\subsection{Areas of Research}

Considering that the main fault zones in the vicinity of the epicenter (Figure 1a) are nearly parallel and mainly distributed along the front of the Himalayas, this paper selects five parallel research areas and analyzes the statistical characteristics of their time processes. In fact, in the analysis of this paper, the time course of each pixel is processed, and finally, the spatial-temporal evolutionary process of the surface temperature is obtained. Here, the selection of the study area mainly aims at the time process analysis of thermal information.

In addition, the range of the study area has an impact on the regional average value, which influences the magnitude of the anomalous changes. The larger the area is, the smaller the amplitude of the anomaly; the smaller the area is, the larger the amplitude of the anomaly. However, the selection of the size of the study area should not affect the changing trend of abnormality. In addition, if this abnormality actually exists, the same results should be obtained from different satellite data. In this paper, two completely independent observations of satellite data are used for comparative studies to verify the reliability of the thermal anomaly information.

\section{Data and Methods}

\subsection{Data}

The information received by the satellites is mainly surface thermal radiation that has been modified by the atmosphere. There are many uncertainties in the direct use of satellite imagery to analyze crustal activity. Atmospheric correction is one of the basic tasks. Land surface temperature, which contributes to surface thermal radiation, can be obtained from satellite remote sensing thermal infrared data after processing, including the atmospheric correction. The data used in this paper are well-known international LST products [39-41]. Therefore, specifically considering atmospheric correction technical issues involved in processing the raw satellite image is not necessary.

The LST products selected in this paper are data from the V5 version of the MODIS/Terra and MODIS/Aqua satellites covering March 2000 or July 2002 to February 2016, which are obtained according to the day/night surface temperature inversion algorithm (Wan et al., 2002; Wan, 2008). These products have a spatial resolution of 0.05 degrees, a time resolution of 8 days, and an accuracy of $1.0 \mathrm{~K}[40]$.

Figure 2 shows the variation in LST with time and its spectral distribution at a specific location (latitude and longitude: $93.7^{\circ} \mathrm{E}, 37.9^{\circ} \mathrm{N}$ ). Figure 2 intuitively shows that the largest change in LST is the annual variation. The annual component represents the influence of solar radiation, and the temperature changes at other frequencies are much weaker than the annual variation. Removing the annual variation is a prerequisite for further identifying abnormal information. 


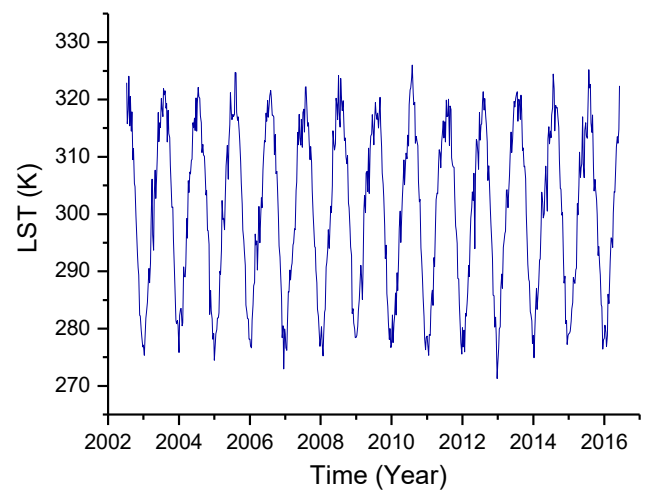

(a)

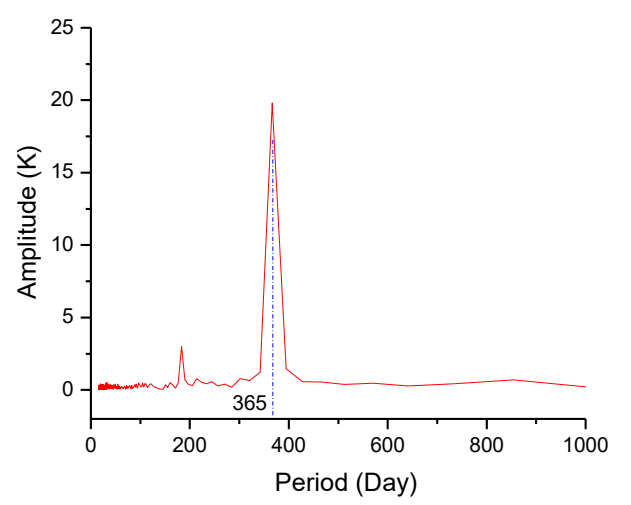

(b)

Figure 2. Variations in land surface temperature (LST) with time (a) and its spectrum distribution (b). Latitude and longitude: $93.7^{\circ} \mathrm{E}, 37.9^{\circ} \mathrm{N}$.

\subsection{Methods}

The change in LST is mainly driven by periodic solar radiation, which provides a periodic heat flux. For the Earth, the Sun is a stable periodic heat source, mainly causing daily and annual changes. The solar-synchronous polar-orbiting satellites, such as Terra and Aqua, pass over the same place only twice in one day, and the daily cycle changes are not observed.

In simple terms, there are the following terms:

$$
T(t, x, y)=T_{0}(x, y)+T_{a}(t, x, y)+\Delta T(t, x, y)
$$

where $T(t, x, y)$ represents the LST with unit of $\mathrm{K}$ or ${ }^{\circ} \mathrm{C}, t$ is time and $x$ and $y$ are spatial coordinates; $T_{0}(x, y)$ is the stable component of the LST, mainly including the influence of terrain and latitude changes; $T_{a}(t, x, y)$ is a stable annual component, representing the influence of solar radiation; and $\Delta T(t, x, y)$ is the residual part, mainly affected by various factors such as atmosphere, vegetation and tectonic activity.

Among these terms, $T_{0}(x, y)$ and $T_{a}(t, x, y)$ are relatively stable and can be considered together. $T_{\text {sta }}(t, x, y)=T_{0}(x, y)+T_{a}(t, x, y) . \quad T_{s t a}(t, x, y)$ represents the strongest component of the LST. Additionally, $T_{s t a}(t, x, y)$ is independent of tectonic activity such as earthquakes. Thus, earthquake anomaly information should be included in $\Delta T(t, x, y)$. For convenience, $\Delta T(t, x, y)$ is called the annual variation residual.

The annual variation residual $\Delta T(t, x, y)$ is selected as the object for further analysis:

$$
\Delta T(t, x, y)=T(t, x, y)-T_{s t a}(t, x, y)
$$

In fact, the annual variation residual $\Delta T(t, x, y)$ is caused by local factors such as crustal activity, human activities and vegetation, and is often disturbed by thermal information from external atmospheric flows. In the above sense, the annual variation residual $\Delta T(t, x, y)$ can be expressed as:

$$
\Delta T(t, x, y)=T_{\text {human }}(t, x, y)+T_{\text {local }}(t, x, y)+T_{\text {atmosphere }}(t, x, y)
$$

Among these terms, $T_{\text {human }}(t, x, y)$ represents the temperature change caused by human activities; $T_{\text {local }}(t, x, y)$ represents the temperature change caused by local factors and is called the local temperature; and $T_{\text {atmosphere }}(t, x, y)$ is the temperature change caused by external heat carried by the atmospheric circulation. $T_{\text {human }}(t, x, y)$ and $T_{\text {atmosphere }}(t, x, y)$ are independent of earthquakes, and earthquake anomaly information should be included in $T_{\text {local }}(t, x, y)$. 
Now, Equation (3) can be written as:

$$
T_{\text {local }}(t, x, y)=\Delta T(t, x, y)-T_{\text {human }}(t, x, y)-T_{\text {atmosphere }}(t, x, y)
$$

In other words, obtaining thermal information related to crustal activity (such as earthquakes) is possible only by extracting the local temperature $T_{\text {local }}(t, x, y)$. In the short term, vegetation and other factors do not change significantly. Therefore, the key is to remove the impact of atmospheric circulation on $\Delta T(t, x, y)$.

From the spatial scale, there are obvious differences in the influence of atmospheric circulation and local factors on LST: the spatial scale of the atmospheric circulation is relatively larger, and the spatial scales of local factors are relatively smaller, especially the linear characteristics of the fault zone, which has obvious one-dimensional features. Thus, Equation (3) can be solved by a two-dimensional spatial analysis method, such as wavelet analysis. Then, $T_{\text {local }}(t, x, y)$ can be obtained by Equation (4). If there is thermal information associated with earthquakes, such information should be found in $T_{\text {local }}(t, x, y)$.

\subsection{Procedure of Data Processing}

Data processing is roughly divided into two categories: time-based and space-based processes. First, for each location $(x, y)$, the surface temperature $T(t, x, y)$ time history is processed to obtain $\Delta T(t, x, y)$. Then, for each time $(t)$, the spatial information regarding $\Delta T(t, x, y)$ is processed to obtain $T_{\text {atmosphere }}(t, x, y), T_{\text {local }}(t, x, y)$ and $T_{\text {human }}(t, x, y)$. Finally, based on $T_{\text {local }}(t, x, y)$, the analysis of the thermal anomaly possibly associated with the Nepal earthquake is carried out. The procedure of data processing is shown in Figure 3.

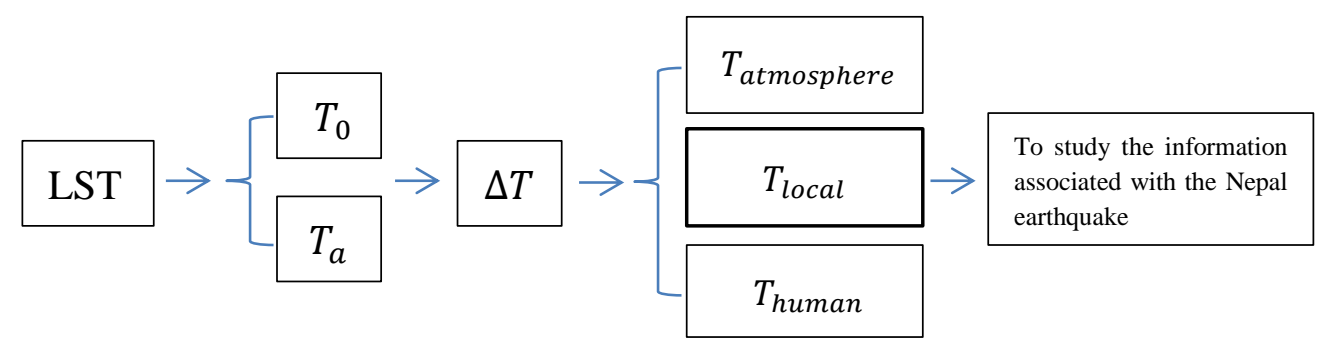

Figure 3. Diagram for the procedure of data processing.

The basic data processing flow is briefly described as follows:

(1) Determine $T_{0}(x, y) . T_{0}(x, y)$ is independent of time and may be obtained through the multiyear average value at location $(x, y)$;

(2) Obtain $T_{a}(t, x, y)$ and $T_{s t a}(t, x, y) . T_{a}(t, x, y)$ is different in different locations, but its time course is determined. For a given location, this value can be obtained from years of data. The specific process includes (i) removing the effects of non-annual components. This removal requires a certain filtering technique to extract the annual variation while filtering out the non-annual components. In this paper, in order to eliminate the influence of non-annual components and the errors introduced by filtering, the one-dimensional orthogonal wavelet theory (Appendix A) is used to extract the annual period components. The advantage of using orthogonal wavelet decomposition is that the extracted annual periodic components do not contain interference by other periodic components [42,43]. (ii) $T_{a}(t, x, y)$ can be obtained by averaging the surface temperatures on the same date from the multiyear data. (iii) According to $T_{0}(x, y)$ and $T_{a}(t, x, y), T_{s t a}(t, x, y)$ is further obtained (Appendix $C$ ).

(3) Calculate $\Delta T(t, x, y) . \Delta T(t, x, y)$ is obtained according to Equation (2), based on $T_{\text {sta }}(t, x, y)$.

(4) Obtain $T_{\text {local }}(t, x, y)$. For a fixed area, the satellite imaging time is short; that is, for the same image, the time in each pixel can be considered constant. At the same time, considering that the spatial scale of the atmospheric circulation is usually large, the spatial decomposition of $\Delta T(t, x, y)$ at each time can be accomplished to obtain $T_{\text {atmosphere }}(t, x, y), T_{\text {local }}(t, x, y)$ and $T_{\text {human }}(t, x, y)$. In this 
paper, the two-dimensional orthogonal wavelet method (Appendix B) is used to spatially decompose $\Delta T(t, x, y) . \Delta T(t, x, y)$ is decomposed into three spatial scales: less than $80 \mathrm{~km} \times 80 \mathrm{~km}$, greater than $600 \mathrm{~km} \times 600 \mathrm{~km}$, and intermediate-scale components. Among these components, those with spatial scales greater than $600 \mathrm{~km}$ are mainly caused by atmospheric circulation, namely, $T_{\text {atmosphere }}(t, x, y)$; the changes caused by human activities are concentrated in spatial components of less than $80 \mathrm{~km}$, namely, $T_{\text {human }}(t, x, y)$. The remaining components represent the contribution of local factors to the LST, namely, $T_{\text {local }}(t, x, y)$.

To avoid the border effect in the spatial decomposition, the spatial scales involved in the above four steps of data processing are larger than those in Figure 2a, as described in Appendix D.

(5) Calculate the variations in thermal information with time, based on $T_{\text {local }}(t, x, y)$. (i) First, according to the study areas (Figure 1), the spatial average results of each study area are separately calculated at each time, so that the time courses of different study areas are obtained. (ii) Then, the statistical parameters, such as the standard mean square error and the histogram of the time process, are calculated separately. Thus, the statistical characteristics of thermal anomaly information that may be related to earthquakes are analyzed further.

\section{Results}

\subsection{Temporal Process}

According to the above procedure, the LST data from the MODIS/Terra and MODIS/Aqua satellites are processed separately. Here, the MODIS/Aqua results are taken as an example for analysis. The MODIS/Terra results are given in the discussion section. In addition, considering that $T_{0}(x, y), T_{a}(t, x, y), T_{\text {human }}(t, x, y)$ and $T_{\text {atmosphere }}(t, x, y)$ are independent of earthquake anomalies, these values are not analyzed (see Appendix D). Here, we focus on the local temperature $T_{\text {local }}(t, x, y)$.

\section{(A) Analysis of standard deviation}

Figure 4 shows the variations in the average local temperature $T_{\text {local }}(t, x, y)$ with time in different study areas. The corresponding statistics for these areas are shown in Table 1 . Table 1 and Figure 4 show that the temperature fluctuations are between -5.83 and $4.61^{\circ} \mathrm{C}$, the fluctuation ranges of the study areas are approximately equivalent, and the fluctuation range is approximately $8 \sim 9{ }^{\circ} \mathrm{C}$; the standard deviation lies between 1.39 and $1.65^{\circ} \mathrm{C}$. Intuitively, there is no obvious increase and decrease. To improve the credibility of the anomaly information, the maximum standard deviation of the five study areas is taken as the reference value, denoted by RefStd. Here, RefStd is $1.65{ }^{\circ} \mathrm{C}$. The double RefStd is $3.30^{\circ} \mathrm{C}$ and is shown as a line in Figure 4a1-a5.

As shown in Figure 4a1-a5, there is a phenomenon in which the temperature changes more than the double RefStd during the Nepal earthquake. However, many times before and after the earthquake, there are occasions when the local temperature value exceeds the double RefStd. Sometimes, the local temperature is higher than the double RefStd, and sometimes, local temperature is lower than the double RefStd. Hence, there is not a unique case in which the temperature changes during the earthquake are more than the double RefStd, which means that the thermal anomaly related to the earthquake simply based on the original local temperature value exceeding the double RefStd is insufficient. 


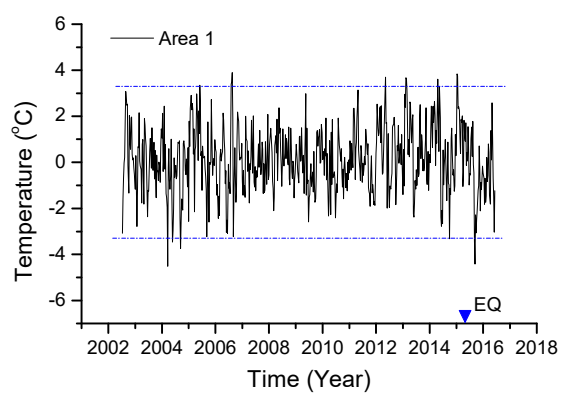

(a1)

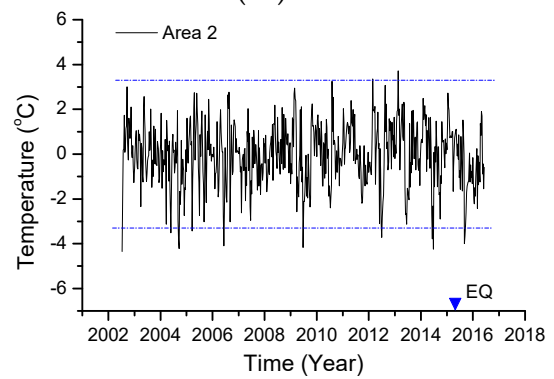

(a2)

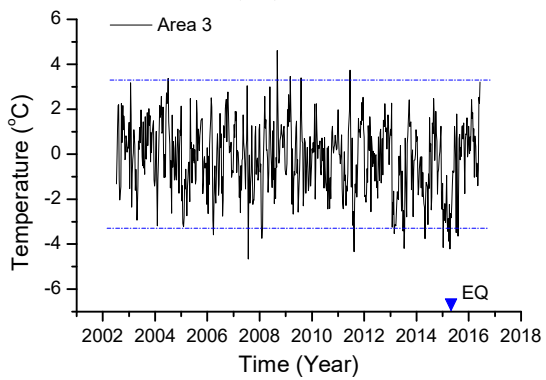

(a3)

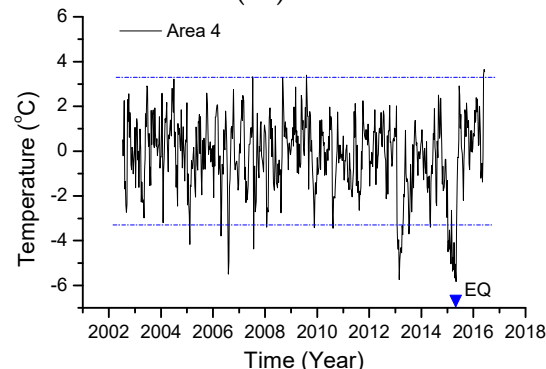

(a4)

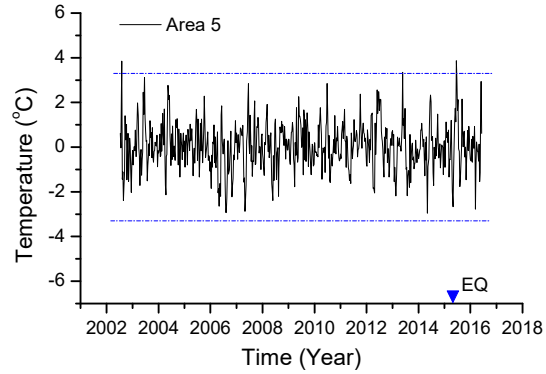

(a5)

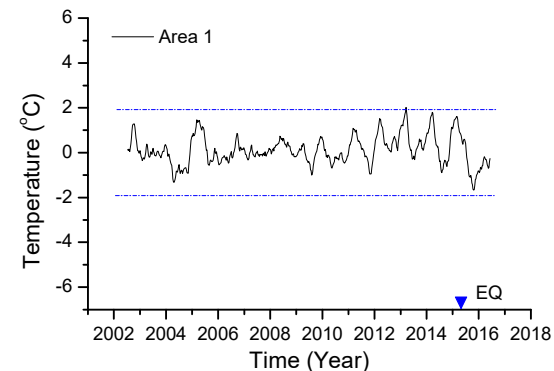

(b1)

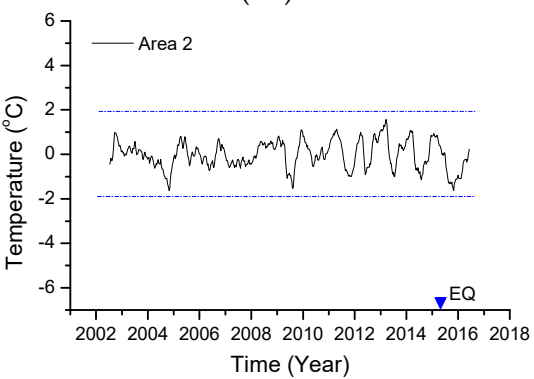

(b2)

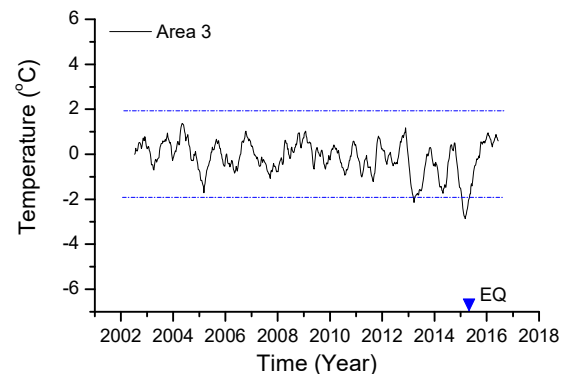

(b3)

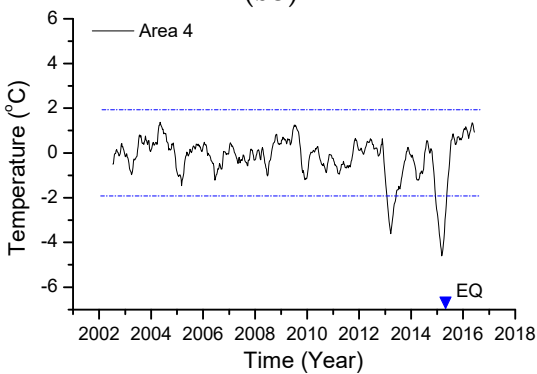

(b4)

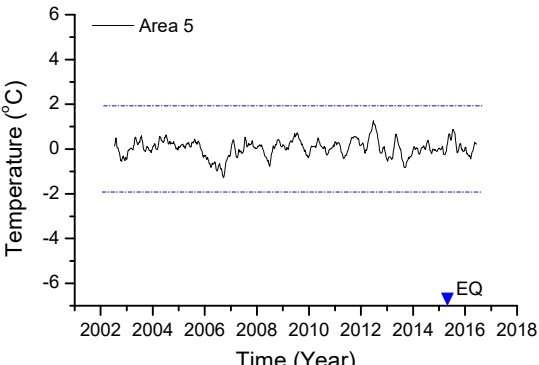

(b5)

Figure 4. Variations in $T_{\text {local }}(t, x, y)$ with time in different study areas. (a1-a5) are the original $T_{\text {local }}(t, x, y)$ values in different study areas; (b1-b5) are the moving averages (the window length is 112 days). The blue lines represent the double value of the maximum standard deviation among study areas. 
Table 1. Statistics for local temperature $T_{\text {local }}(t, x, y)$.

\begin{tabular}{cccccccccc}
\hline \multirow{2}{*}{ Study Area } & \multirow{2}{*}{ Totals } & \multicolumn{3}{c}{ Non-Smoothed } & \multicolumn{4}{c}{ Smoothed } \\
\cline { 3 - 9 } & & Mean & Std $^{\text {a }}$ & Max & Min & Mean & Std & Max & Min \\
\hline No. 1 & 641 & 0.11 & 1.42 & 3.91 & -4.51 & 0.11 & 0.64 & 2.02 & -1.66 \\
No. 2 & 641 & -0.03 & 1.39 & 3.71 & -4.35 & -0.02 & 0.62 & 1.57 & -1.63 \\
No. 3 & 641 & -0.17 & 1.55 & 4.61 & -4.66 & -0.17 & 0.75 & 1.37 & -2.86 \\
No. 4 & 641 & -0.20 & 1.65 & 3.64 & -5.83 & -0.20 & 0.95 & 1.37 & -4.60 \\
No. 5 & 641 & 0.11 & 1.42 & 3.91 & -4.51 & 0.04 & 0.37 & 1.27 & -1.28 \\
\hline
\end{tabular}

a: Standard deviation of time-series data; Note: the unit of local temperature is ${ }^{\circ} \mathrm{C}$.

(B) Analyses of moving average

Further, as shown in Figure 4a1-a5, the cases when these temperature values exceed the double RefStd are mostly short-term changes. Considering that the meteorological factors are extremely complicated, these factors are usually short-term temperature changes of a few or even a dozen ${ }^{\circ} \mathrm{C}$ per day. Information related to earthquakes can easily be submerged in meteorological changes. To suppress the influence of short-term changes and to highlight the earthquake-related information, the temperature change signals of the above different study areas are smoothed over a long time. In this paper, taking the 112-day moving average window weakens the impact of short-term changes. The results are shown in Figure 4b1-b5, and the relevant statistical information is given in Table 1.

After smoothing, the temperature fluctuation is between -4.6 and $2.02{ }^{\circ} \mathrm{C}$; the standard deviation is significantly reduced to between 0.37 and $0.95{ }^{\circ} \mathrm{C}$. Similarly, the maximum standard deviation of the five areas is $0.95^{\circ} \mathrm{C}$, which is taken as the reference value, denoted as SRefStd. The lines in Figure $4 \mathrm{~b} 1-\mathrm{b} 5$ represent the double SRefStd. As shown in Figure 4b1-b5, only study areas 3 and 4 are more than the double SRefStd (Figure 4b3,b4), and both were cooling before the earthquake. Among these areas, the strongest deviation corresponds to the occurrence of the earthquake.

In short, from the time process, there was significant cooling before the earthquake, and the temperature reached the lowest value during the earthquake and returned to normal after the earthquake. The variation range was more than twice the SRefStd, and the change in area 4 reached $-4.60^{\circ} \mathrm{C}$, even more than 4 times the SRefStd. That is, in study areas 3 and 4 , a change in temperature more than the normal background occurred before the earthquake.

\section{(C) Frequency distribution}

According to the previous analysis, the abnormal change in area 4 is the most obvious (Figure 4b4), while the adjacent study area 5 has no obvious abnormality (Figure 4b5). Here, study area 4 and area 5 are selected for further statistical analysis of the frequency distribution to compare the similarities and differences in temperature distributions with and without abnormalities.

Figures $4 \mathrm{~b}$ and $5 \mathrm{a}$ show the frequency distribution of the smoothed temperature values of study area 4 and area 5 (Figure $4 \mathrm{~b} 4, \mathrm{~b} 5$ ). Figure 5 illustrates that (i) in the range of $\left[-1.5^{\circ} \mathrm{C}, 1.5^{\circ} \mathrm{C}\right]$, the distributions of the two study areas are similar and should be close to the same distribution pattern (Figure $5 \mathrm{a}, \mathrm{b}$ ); (ii) in the range of $\left[-3.0^{\circ} \mathrm{C},-1.5^{\circ} \mathrm{C}\right]$, there is another temperature distribution pattern for study area 4 , which is close to a uniform distribution (Figure 5c). That is, the temperature distribution of study area 4 includes a temperature distribution pattern different from that of study area 5 . We simply decompose the temperature distribution contained in study area 4 into two cases, close to the average distribution and the normal distribution, as shown in Figure $5 c$,d. Given these two distributions, for the distribution shown in Figure $5 \mathrm{c}$, the value in $\left[-3.0^{\circ} \mathrm{C},-1.5^{\circ} \mathrm{C}\right]$ is the same as that shown in Figure $5 \mathrm{a}$, and the value in $\left[-1.5^{\circ} \mathrm{C}, 0^{\circ} \mathrm{C}\right]$ is the mean of $\left[-3.0^{\circ} \mathrm{C},-1.5^{\circ} \mathrm{C}\right]$. The distribution pattern in Figure $5 \mathrm{~d}$ is similar to that in Figure $5 \mathrm{~b}$. The distribution shown in Figure $5 \mathrm{c}$ reflects the temperature distribution of the difference between study areas 4 and 5, and the difference is mainly reflected in the low-temperature part. This result is consistent with the characteristics of the cooling for the time course performance. 


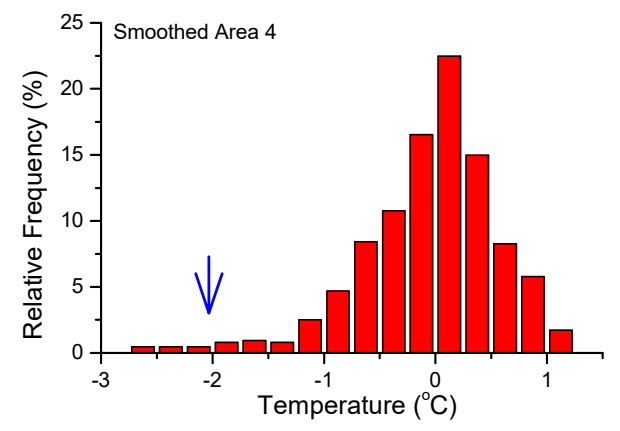

(a)

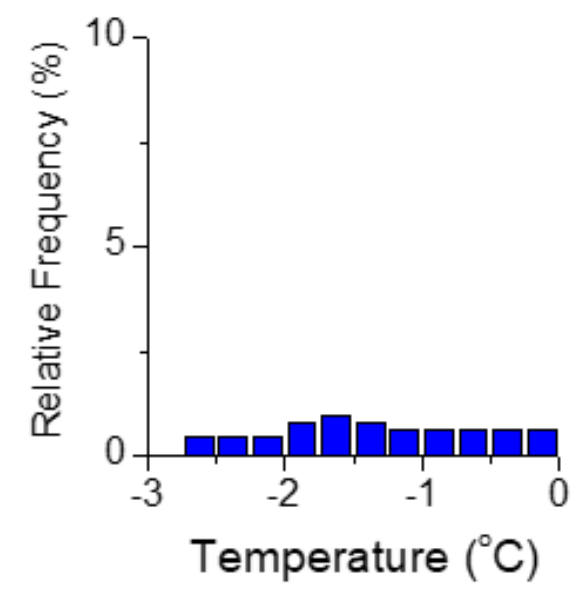

(c)

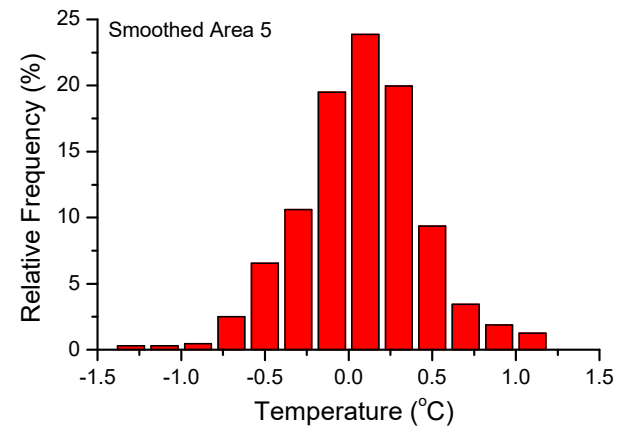

(b)

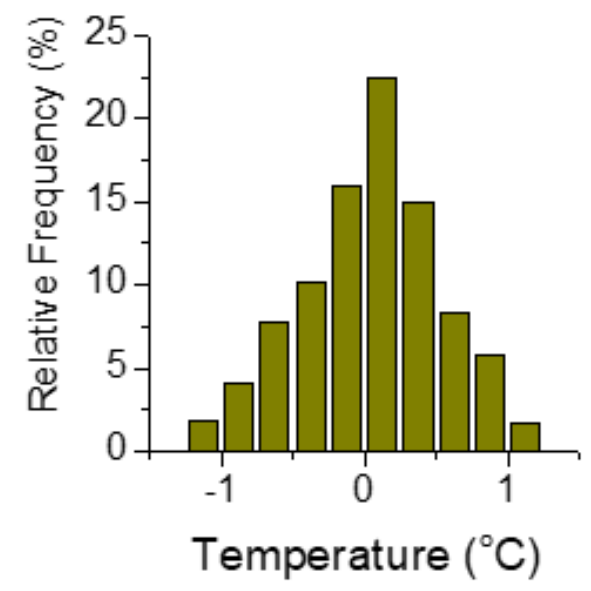

(d)

Figure 5. The frequency distribution of the smoothed $T_{\text {local }}$ in study area 4 (a) and area 5 (b). The distribution in (a) can be simply decomposed into the two distributions in (c) and (d).

\subsection{Spatial Distribution}

Figure 6 shows the spatial distribution of the local temperature $T_{\text {local }}(t, x, y)$ before and after the Nepal earthquake. In Figure 6, the most obvious feature is the apparent temperature decrease near the epicenter at the time of the earthquake (Figure $6 \mathrm{~b}$ ), referred to as coseismic cooling. The coseismic cooling is mainly distributed in study area 3 and area 4 . After the earthquake, the cooling strip is obviously weakened. The coseismic cooling strips are distributed along the Himalayas and are approximately $1300 \mathrm{~km}$ long. The widths of the East and West sides of the coseismic cooling strips are slightly different, with an average temperature decrease of $5.6^{\circ} \mathrm{C}$. The Western section (AB) is approximately $90 \mathrm{~km}$ wide and $500 \mathrm{~km}$ long, and the East side (BC) is approximately $190 \mathrm{~km}$ wide and $800 \mathrm{~km}$ long.

In comparing the spatial distributions of local temperature $T_{\text {local }}(t, x, y)$ before and during the earthquake, the abovementioned cooling strip ( $\mathrm{AB}$ section) appeared on the West side of the earthquake before the earthquake occurred (Figure 6a). That is, the evolution of the cooling strip was from West to East, which is consistent with the extension of the coseismic rupture from West to East [29].

Further, there is still another important phenomenon: from the perspective of seismic location, the epicenter of the Nepal earthquake is approximately 40 kilometers away from the cooling strip (Figure 6b). In fact, the earthquake rupture has a certain length, and the epicenter refers to the rupture starting point. According to the seismic wave inversion results, the coseismic rupture extends $\sim 80 \mathrm{~km}$ to the North and $\sim 100 \mathrm{~km}$ Southeast [36]. Since the earthquake occurred on a low-angle thrust fault $\left(\sim 15^{\circ}\right)$, the distance extended to depth is projected to the North, which is equivalent to a Northward extension of $\sim 80 \mathrm{~km}$. That is, the stopping boundary of the rupture is already in the cooling strip. In fact, as shown in Figure 1a, some of the aftershocks that occurred on 25 April 2015 were located in the 
study area 3, namely in the cooling strip. Therefore, although the earthquake epicenter is outside the cooling zone, there is a spatial correlation between the cooling zone and the coseismic rupture during the Nepal earthquake.
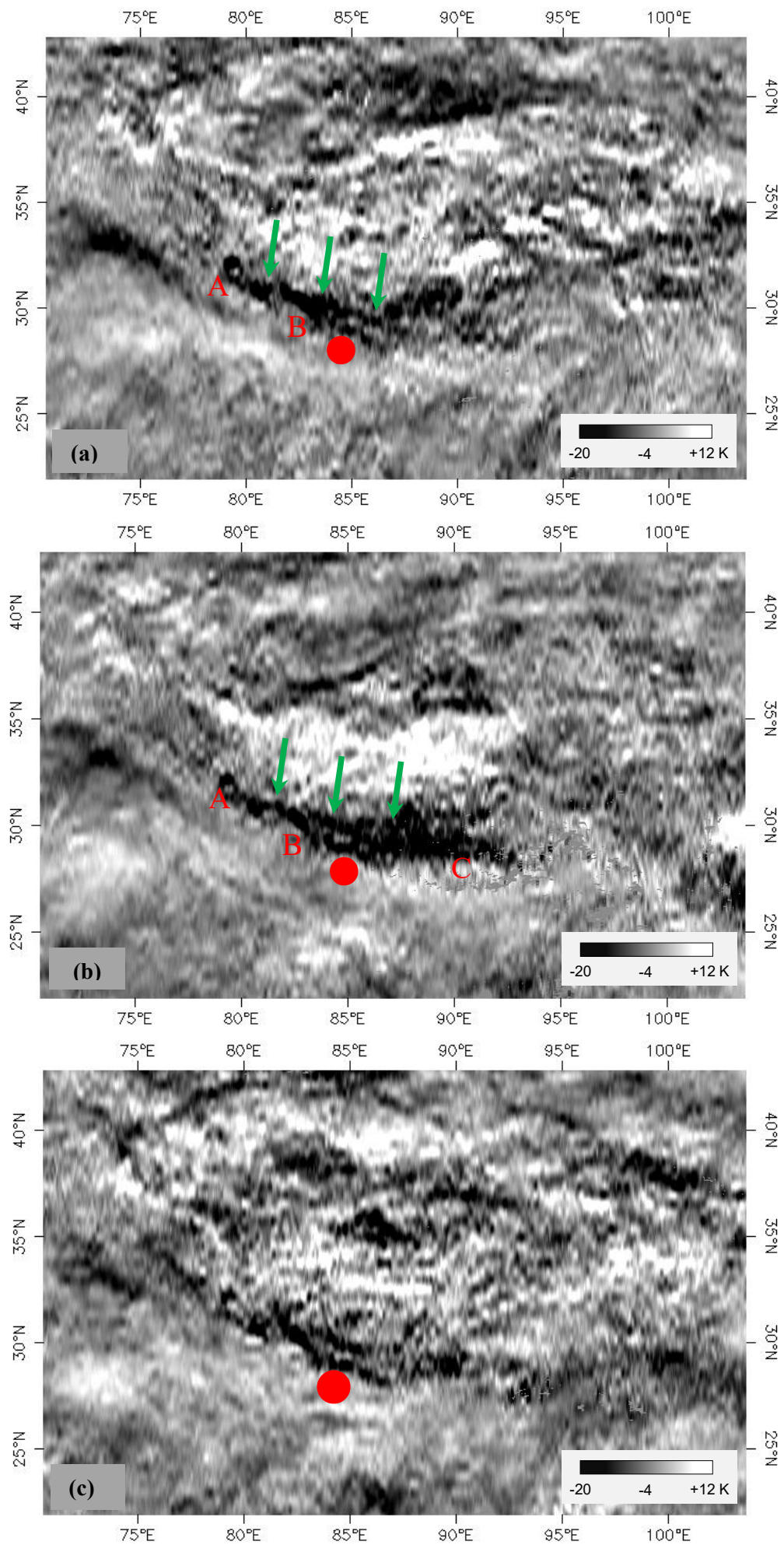

Figure 6. Spatial distributions of local temperature $T_{\text {local }}$ before and after the Nepal earthquake (Aqua). (a-c) are pre-earthquake (15-22 April 2015), coseismic (23-30 April 2015) and postearthquake (31 April-7 May 2015), respectively. The red solid cycle is the Nepal earthquake. 
In summary, a significant cooling zone is found in the local temperature field $T_{\text {local }}(t, x, y)$. Moreover, the coseismic cooling zone is associated with the coseismic rupture distribution, and the evolution direction is highly consistent with that of coseismic rupture. This feature implies that the cooling zone might be related to the evolutionary process of crustal stress.

\section{Discussion}

\subsection{Comparison of Two Cooling Events before the Earthquake}

As shown in Figure 4b4, two significant cooling events occurred during the time course in study area 4, which are further analyzed here. For convenience, they are labeled S1 and S2, as shown in Figure 7. S1 was a relatively sharp decrease in temperature. From the normal background to the valley point, $\mathrm{S} 1$ experienced approximately 40 days in which the temperature decrease was $\sim 6^{\circ} \mathrm{C}$; then, the temperature increased, and the recovery duration to normal level was close to the decrease duration. During these days, the stage of fastest decrease lasted approximately 16 days. Meanwhile, S2 showed a slow decrease. From the normal background to the valley point, S2 lasted 320 days, and the temperature decrease was $\sim 7^{\circ} \mathrm{C}$. S2 reached the minimum value at the occurrence of the earthquake and recovered to the normal background value soon after the Nepal earthquake.

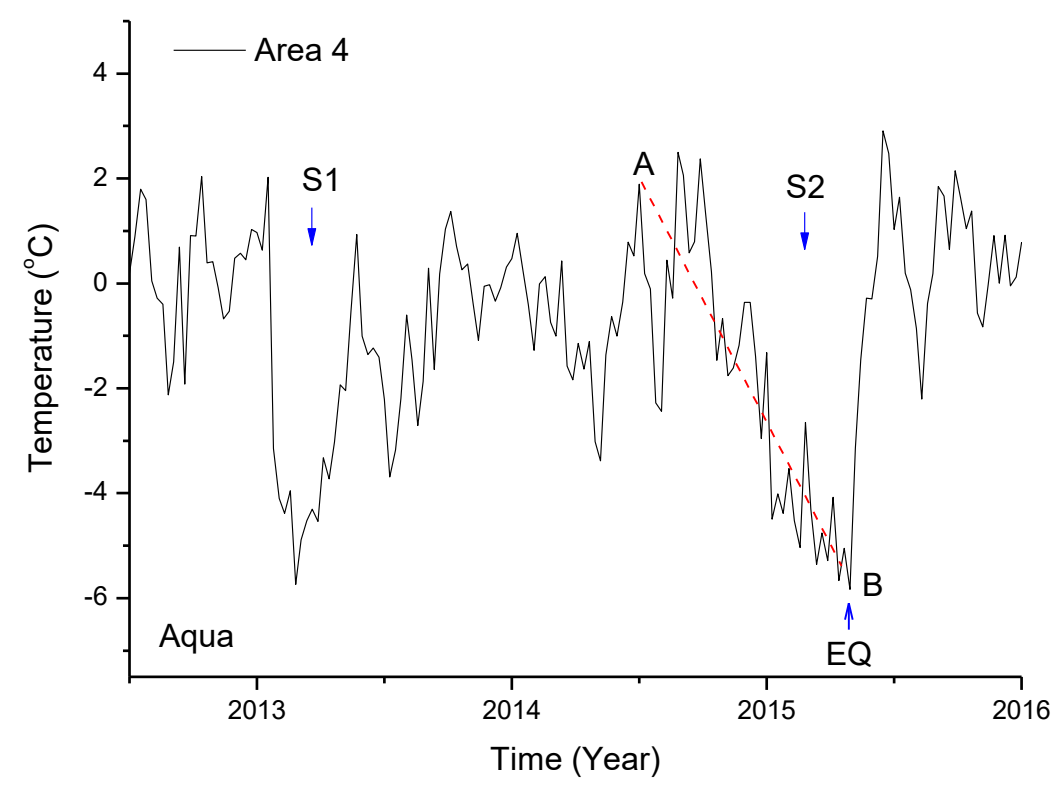

Figure 7. Two cooling events in local temperature $T_{\text {local }}$ in study area 4 before the Nepal earthquake (Aqua satellite).

Although both S1 and S2 are cooling events, there is a significant difference in duration from the normal background to the minimum point. The duration of S1 lies within the range of the duration of weather events. The magnitude of S1 is more than twice the standard deviation (Figure $4 \mathrm{a} 4$ or Figure $4 \mathrm{~b} 4$ ); however, confirming that $\mathrm{S} 1$ was uniquely generated by crustal activity is hard, as its duration is short and might be easily mixed by the effect of weather events (i.e., continuous rainfall or snow).

As for S2, the temperature decrease lasted for nearly a year, and can hardly be interpreted as an event associated with meteorological changes. Particularly, the temperature reached a minimum value when the earthquake occurred and quickly increased back to normal background after the earthquake. This trend of change largely coincided with the occurrence of the earthquake, which implies that the change was related to the earthquake. 


\subsection{Comparison of Data from Different Satellites}

The results from the MODIS/Aqua satellite are given above. For comparative analysis, we also calculated the LST data from the MODIS/Terra satellite. The calculation process is consistent with that for the Aqua satellite, and the results are given in Figure 8. Figure 8a shows the spatial distribution of the coseismic response according to the MODIS/Terra satellite. As illustrated in Figure 8a, a clear coseismic cooling strip appears. Comparing Figure $6 \mathrm{~b}$ with Figure $8 \mathrm{a}$, the spatial distribution of the coseismic cooling zone for the Terra satellite is seen to be basically consistent with that for the Aqua satellite.

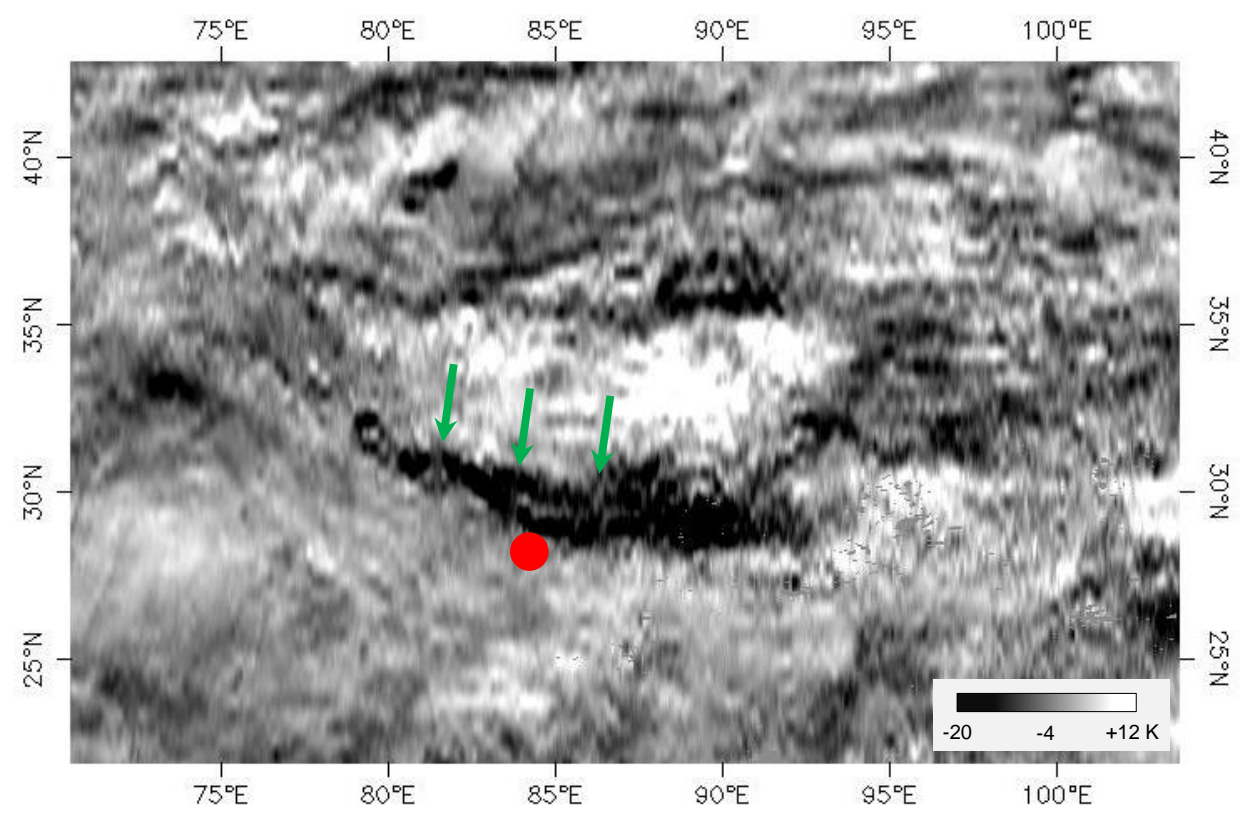

(a)

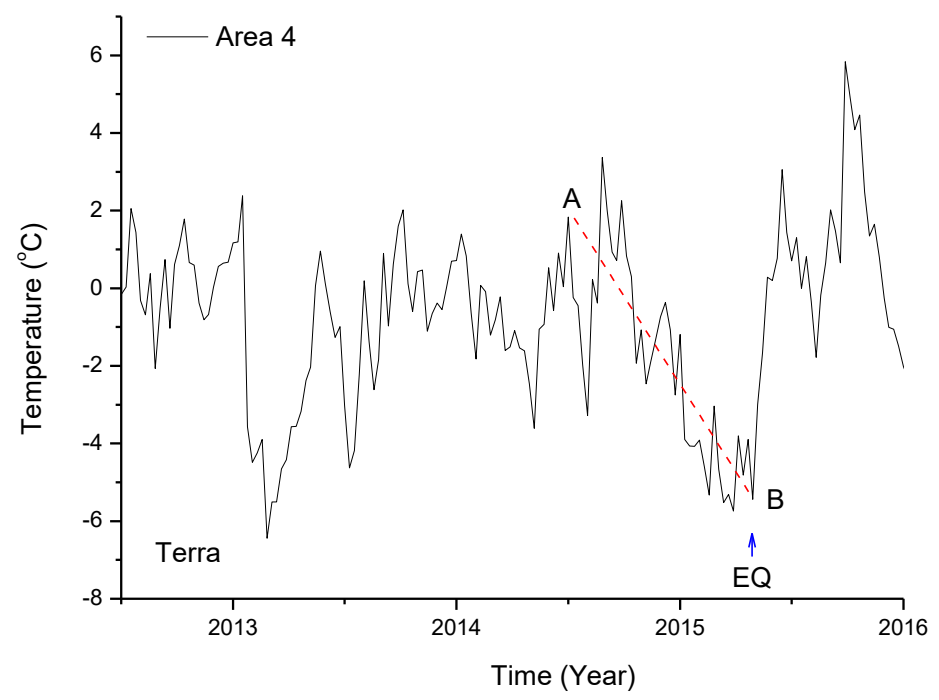

(b)

Figure 8. The case of the Terra satellite. (a) Spatial distribution of coseismic temperature (23-30 April 2015); (b) the temporal process of local temperature in study area 4 . AB in panel (b) represents the cooling process before the earthquake, and EQ is the Nepal earthquake. The red solid cycle is the Nepal earthquake. 
Among the above-mentioned results for the Aqua satellite, study area 4 is located in the main cooling zone, and Figure $8 \mathrm{~b}$ also shows the change in local temperature over time in study area 4 before and after the Nepal earthquake. Figure $8 \mathrm{~b}$ illustrates that before the earthquake, study area 4 showed continuous cooling $(\mathrm{AB})$, which reached a low value near the time of the earthquake, and a rapid increase in temperature back to the normal background after the earthquake. Comparing Figure 7 with Figure $8 \mathbf{b}$, the results for the two satellites Terra and Aqua are seen to be basically the same for the time course of study area 4 .

In summary, for both the temporal process before and after the earthquake and the spatial distribution, the results obtained from the Terra satellite are basically consistent with those from the Aqua satellite. The data from different satellites yield the same results, which indicate that the cooling phenomenon is independent of satellites. In other words, the phenomenon is repeatable and reliable.

\subsection{Physical Mechanism}

There are several mechanisms that cause thermal infrared anomalies [23], mainly including deflation of gases such as $\mathrm{CO} 2$ and $\mathrm{CH} 4$ within the crust [9], p-hole excitation theory (Freund, 2007a, b), conversion between mechanical and thermal energy [22,44,45], and systematic coupling effects, such as seismic-atmosphere-ionosphere coupling $[16,46]$. Most physical mechanisms are associated with stress changes or their secondary effects, but each mechanism emphasizes different aspects of physical properties that are produced by or accompany stress changes, such as gas, electricity, magnetism or heat. In general, each physical mechanism itself has an experimental basis, and focuses on analysis of a conceptual model, which is helpful for establishing a qualitative understanding of satellite thermal infrared anomalies.

The cooling phenomenon found in this paper was also found in another thrusting-type earthquake, namely the Wenchuan Mw7.9 earthquake, which occurred in Longmenshan thrust faults located at the Eastern boundary of the Qinghai-Tibet Plateau [27]. Additionally, there are some other cases of decreasing temperature anomalies [47]. Freund's p-hole theory $[18,19]$ is widely used as the physical basis of why a temperature rise can be observed, but for such a strong temperature drop as the ones reported by the present study and the other cases of decreasing temperature anomalies [27,47], there is not any physical basis explained, and this requires us to explore the other physical mechanism.

According to theoretical and experimental studies, temperature has a sensitive response to stress changes, and the temperature decreases when the extensional stress enhances [48-51]. So, there exists the possibility of the temperature drop as stress relaxation is generally accompanied by the occurrence of earthquakes. The Nepal earthquake was a thrust-faulting earthquake, and stress relaxation occurred on the upper side of the thrust fault (namely on the Northern side of the Nepal earthquake). The coseismic temperature decrease is qualitatively in accordance with the coseismic stress drop on the upper side of the thrust fault. As mentioned above, the values for the stress drops of the Nepal earthquake are highly dependent on the model [37]. Now, we cannot give more interpretation. Frankly, we cannot say that this explanation is final; however, we hope that it can provide some clues for approaching a reasonable physical interpretation in the future.

\subsection{Implication of the Coseismic Temperature Response}

Since the physical model is insufficient, if certain information from the process of thermal information change can be found, such information can be helpful to further understand the phenomenon. The following assumption seems to be plausible: for an earthquake-related signal, if there is an abnormality before the earthquake, the peak should be reached at the time of occurrence of this earthquake and should weaken after the earthquake. The factors that influence LST are extremely complicated; in particular, meteorological changes occur rapidly. Thermal information related to earthquakes might be overwhelmed by complex meteorological effects. In general, the coseismic response should be a relatively certain piece of information that may be used as a basis for determining whether other information is related to an earthquake. 
Through the above analysis, during the Nepal earthquake, a cooling strip corresponding to the seismogenic structure was found. In particular, from the temporal perspective, the temperature slowly decreased before the earthquake, reached a minimum at the time of occurrence of the earthquake, and returned to a normal level after the earthquake. This sequence indicates that this spatial and temporal change is closely related to the earthquake and may have been caused by the tectonic activity associated with the Nepal earthquake.

In addition, as mentioned above, the variation range of this signal was more than twice the maximum standard deviation among study areas (Figure 4). Meanwhile, there exists the physical possibility that the temperature drop with the stress relaxation is generally accompanied by the occurrence of an earthquake. Lastly, considering that these changes were related to and initiated before the earthquake, they might even have been potential precursors of the Nepal earthquake.

\section{Conclusions}

In this paper, a total of 15 or 17 years of MODIS/Aqua and MODIS/Terra satellite remote sensing LST products from 2002 or 2000 to 2016 was selected to analyze the temperature change before and after the Mw 7.9 earthquake in Nepal on 25 April 2015 and to explore possible thermal information related to earthquakes. A set of "physical" methods was used to analyze the effects of non-seismic factors such as solar radiation and atmospheric circulation. The results from the two satellites are listed below:

(1) From the time course, there was significant cooling before the earthquake in study areas 3 and 4, which reached the lowest values during the earthquake and returned to normal after the earthquake. The variation range was more than twice the maximum standard deviation among study areas, and even more than 4 times the maximum standard deviation among study areas. That is, in study areas 3 and 4 , there occurred changes in temperature greater than normal backgrounds before the earthquake. This cooling peaked at the time of the earthquake and returned to normal after the earthquake. This sequence indicates that changes closely related to the earthquake over time may have been due to tectonic activity associated with the Nepal earthquake. Considering that these changes occurred before the earthquake, they may even have been precursors to the Nepal earthquake.

(2) From the spatial distribution, there were cooling strips corresponding to the seismogenic structure during the earthquake. These coseismic cooling strips were distributed along the Himalayas and were approximately $1300 \mathrm{~km}$ long. The widths of the East and West sides were slightly different, with an average temperature decrease of $5.6^{\circ} \mathrm{C}$. The Western section (AB) was approximately $90 \mathrm{~km}$ wide and approximately $500 \mathrm{~km}$ long; the East side (BC) was approximately $190 \mathrm{~km}$ wide and $800 \mathrm{~km}$ long. The Western side of the cooling strips appeared before the earthquake.

(3) From the relationship between the stress change and the temperature response, if possible, the above temperature change process might indicate that there was a progression in which the crustal stress gradually decreased before the earthquake, which is consistent with the coseismic stress drop of this thrust-type earthquake.

Author Contributions: Conceptualization, S.C., P.L. and L.C.; methodology, S.C. and P.L.; software, S.C. and P.L.; validation, S.C., P.L. and L.C. and Z.J.; formal analysis, S.C.; investigation, S.C., T.F., D.W., Z.X., and G.Z.; resources, X.X.; data curation, Z.J.; writing — original draft preparation, S.C.; writing-review and editing, S.C., L.C., and Z.J.; visualization, L.C.; supervision, P.L.; project administration, S.C., T.F. and D.W.; funding acquisition, S.C., T.F. and D.W. All authors have read and agreed to the published version of the manuscript.

Funding: This research was funded by the National Key R\&D Program of China (Grant No. 2018YFC1503304), the Basic Research Funds from the Institute of Geology, China Earthquake Administration (Grant No. IGCEA1605) and Project of China Railway (Grant No. 2017G008-F, KYY2018117 (18-19)) and the National Natural Science Foundation of China (Grant No. 41474162).

Acknowledgments: We thank NASA's Land Processes Distributed Active Archive Center (DAAC) and the MODIS Land Surface Temperature Group, from ICESS, University of California, Santa Barbara, for supplying the LST products. The work was done under the leadership of Jin Ma who passed away on 12 August 2018 after the manuscript had been finished. Thanks are owed to Yongsheng Zhou and Changrong He who provided helpful 
support for this work. We also thank three anonymous reviewers for their critical reviews of an earlier version of the manuscript and two anonymous reviewers for their reviews of the paper.

Conflicts of Interest: The authors declare no conflict of interest.

\section{Appendix A. One-Dimensional Wavelet Analysis}

Wavelet transformation is a theory that has been widely applied in mathematics and other fields of science and technology since the end of the 20th century [52,53]. The best feature is the auto-adaptability and mathematical microscope. This theory can automatically adjust relevant parameters according to the object being studied and elucidate time-frequency local properties. Wavelet analysis is derived from traditional Fourier analysis but avoids the limitation that Fourier analysis cannot yield local information, and wavelet analysis can provide spectral characteristics of signals over time [54].

For a given wavelet function $h(x)$, a family of functions is obtained by expansion and translation [52]:

$$
h_{j, k}(x)=2^{j / 2} h\left(2^{j} x-k\right)
$$

where $j$ is the scaling factor and $\mathrm{k}$ is the translation factor. The smaller the scale is, the higher the frequency; the larger the scale is, the lower the frequency. The wavelet transform at any scale is:

$$
C(j, k)=<h_{j, k}, f>=2^{j / 2} \int \overline{h_{j, k}(x)} f(x) d x
$$

where $C(j, k)$ is the wavelet coefficient and $h_{j, k}(x)$ is the conjugate of $h_{j, k}(x)$.

For the square integral function space $\overline{L^{2}(R)}$, any given signal $f(x) \in L^{2}(R)$ can be decomposed into different frequency bands by scale [52,53]:

$$
\begin{aligned}
f(x) & =\sum_{j, k=-\infty}^{\infty} C(j, k) h_{j, k}(x) \\
& =\cdots+g_{-1}(x)+g_{0}(x)+g_{1}(x)+\cdots+g_{j}(x)+\cdots
\end{aligned}
$$

where $g_{j}(x)$ is the component of $f(x)$ on scale $j$.

If $\mathrm{h}$ is an orthogonal wavelet, the components in (A4) are orthogonal to each other, namely:

$$
<g_{i}(x), g_{j}(x)>=0(i \neq j)
$$

where $<g_{i}(x), g_{j}(x)>$ represents the inner product of the two components, and if the inner product is zero, the components are uncorrelated and can be added in any combination without being affected by other components. Selecting the appropriate frequency band $g_{j}(x)$ can be accomplished by combining Equation (A4) with Equation (1).

In theory, Equation (A4) can be infinitely decomposed, but in fact, $\mathrm{j}$ cannot be too large; only a limited number of wavelet coefficients can be processed. To accurately calculate these coefficients, the size of $\mathrm{j}$ should be appropriate [54]. In this paper, the orthogonal wavelet Coiflet is used for decomposition. The appropriate decomposition number corresponding to the wavelet Coiflet is 5 , which is the number of decomposition layers in this paper.

\section{Appendix B. Two-Dimensional (2D) Wavelet Analysis}

Two-dimensional (2D) analysis is a common problem in signal processing, such as image processing. Two-dimensional wavelet analysis is one of the important methods of two-dimensional signal processing [42].

Assuming a signal function $f(x, y) \in L^{2}\left(R^{2}\right), \varphi(x, y)$ is a two-dimensional mother wavelet function whose construction can be formed by the tensor product of the one-dimensional mother wavelet or by the nontensor product method, $\varphi_{a, b, c}(x, y)=|a|^{2} \varphi\left(\frac{x-b}{a}, \frac{x-c}{a}\right), b, c \in R, a \in R-\{0\}$. 
The two-dimensional continuous wavelet transform is:

$$
W f(a, b, c)=\operatorname{CWT}(a, b, c)=|a|^{-1} \iint f(x, y) \varphi\left(\frac{x-b}{a}, \frac{y-c}{a}\right) d x d y
$$

If we set $a=2^{-j}, b=k_{1} 2^{-j}$, and $b=k_{2} 2^{-j}, j, k_{1}, k_{2} \in Z$, the two-dimensional discrete wavelet transform is:

$$
D W T\left(j, k_{1}, k_{2}\right)=2^{j} \sum_{l_{2}} \sum_{l_{1}} f\left(l_{1}, l_{2}\right) \varphi\left(2^{j} l_{1}-k_{1}, 2^{j} l_{2}-k_{2}\right)
$$

where $l_{1}, l_{2} \in Z$.

Assume that the dual wavelet corresponding to the two-dimensional mother wavelet $\varphi(x, y)$ is $\underline{\varphi}(x, y), \underline{\varphi}_{a, b, c}(x, y)=|a|^{2} \underline{\varphi}\left(\frac{x-b}{a}, \frac{x-c}{a}\right)$. Then, there is a unified reconstruction equation corresponding to the two-dimensional wavelet transform, namely, the inverse wavelet transform:

$$
f(x, y)=<f(x, y), \varphi_{a, b, c}>\bar{\varphi}_{a, b, c}=<f(x, y), \bar{\varphi}_{a, b, c}>\varphi_{a, b, c}
$$

where $<,>$ represents the inner product of the two functions. The discrete case represents the sum of the product of the corresponding components.

According to the two-dimensional multiresolution analysis theory and the Mallat algorithm (Mallat, 1989), a two-dimensional function $f(x, y)$, after a two-dimensional wavelet decomposition, can be decomposed into a sum of a smooth component and three detail components. After being decomposed by the J layer, there is the following:

$$
f(x, y)=A_{-J} f+\sum_{j=-1}^{-J} \sum_{e=1}^{3} D_{j}^{e} f
$$

where $J$ is a positive integer, $A_{-J} f$ represents the smoothed component after the $J$-layer decomposition and $D_{j}^{e} f$ represents the $e$-th detail component of the $j$-th layer decomposition. The above equation is a multiresolution decomposition equation for two-dimensional wavelets, namely, the Mallat algorithm [43].

In Equation (A9), the various components obtained by the decomposition of the J layer are essentially the decomposition of the time-frequency space. If the orthogonal wavelet is used to complete the above decomposition, the components are orthogonal to each other, and the signals are independent of each other. According to the previous analysis, there is a significant spatial scale difference between the atmospheric circulation and the in situ temperature in Equation (3). That is, Equations (3) and (A9) are similar. The wavelet multiresolution analysis represented by Equation (A9) can separate the atmospheric circulation and the in situ temperature components in the annual variation. From the LST field, the in situ temperature component generated by the local factor is extracted, and information on crustal activity can be found. This process is also a new application for wavelet analysis.

\section{Appendix C. The Stable Annual Variation $\left(T_{\text {sta }}\right)$ in Land Surface Temperature (LST)}

Since both T0 and Ta are stable in LST, they can be merged as a stable annual variation field (SAVF), Ta. SAVF is the main component of LST and can be taken as the background because solar radiation is the factor with the strongest influence (Figure A1).

SAVF can be obtained from the LST product by the following steps [55]: (1) Remove the influence of non-annual components with the help of a curtain method for filter waves. Meanwhile, the annual signal should be retrieved. Wavelet analysis is applied to retrieve the annual component in order to reduce the influence of other periodic signals and errors produced by filtering, with annual periodicities in the range of 256-512 days. One of the advantages of the orthogonal wavelet method is that the retrieved annual periodic components barely experience interference by other components $[42,43]$. 
(2) Obtain SAVF by averaging the annual temperature signals for the same calendar days. As shown in Figure A1, SAVF is similarly sinusoidal, which agrees with the solar radiation during Earth's revolution.
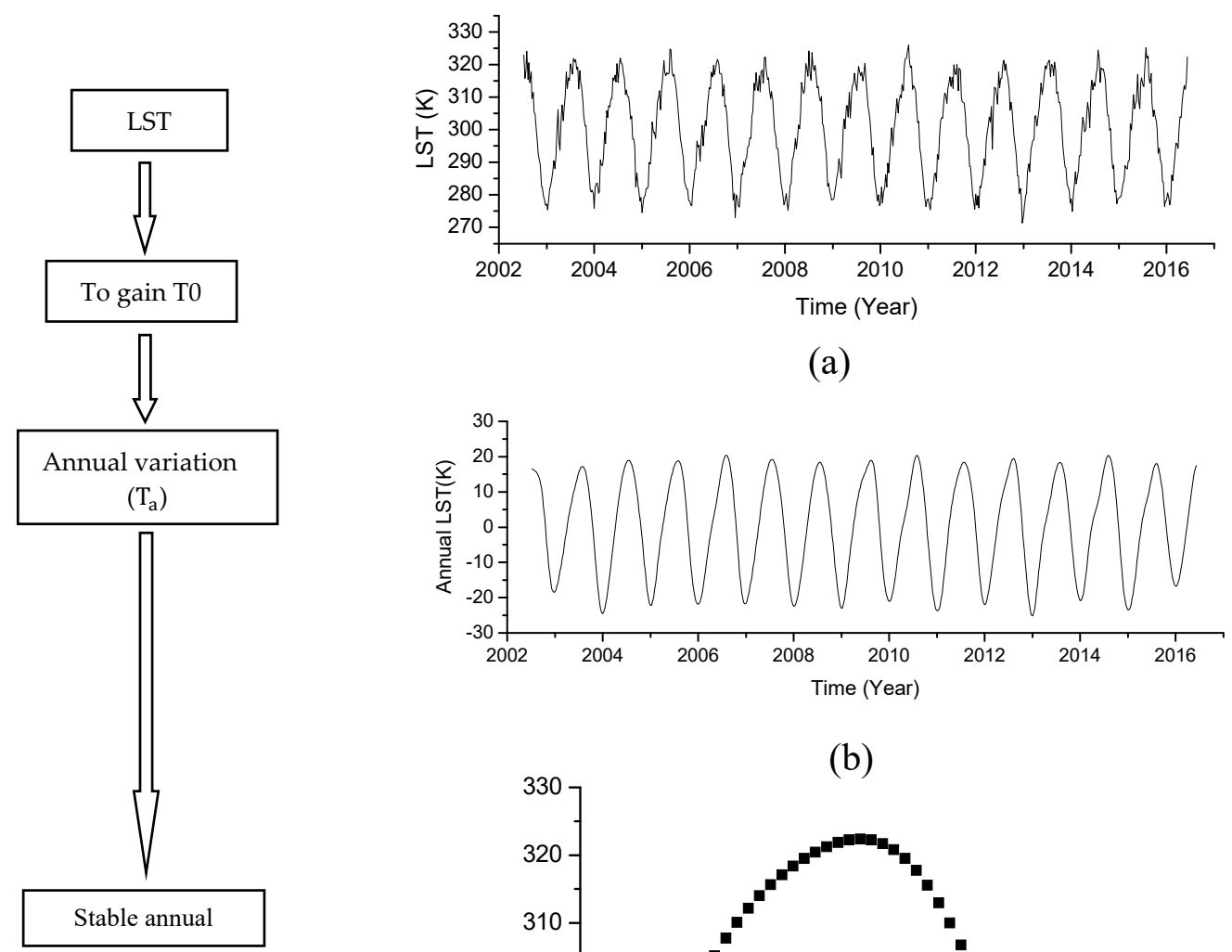

(a)

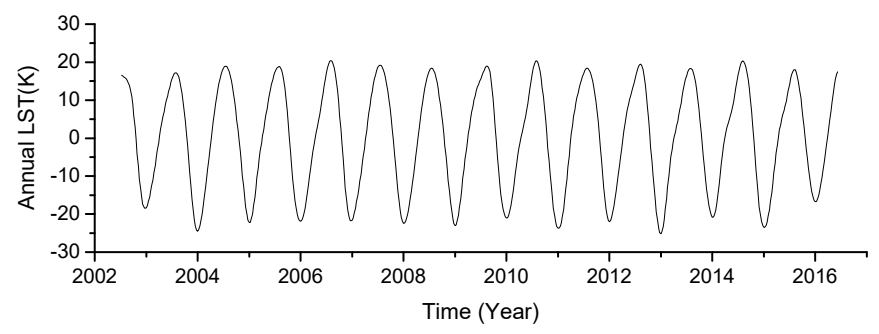

(b)

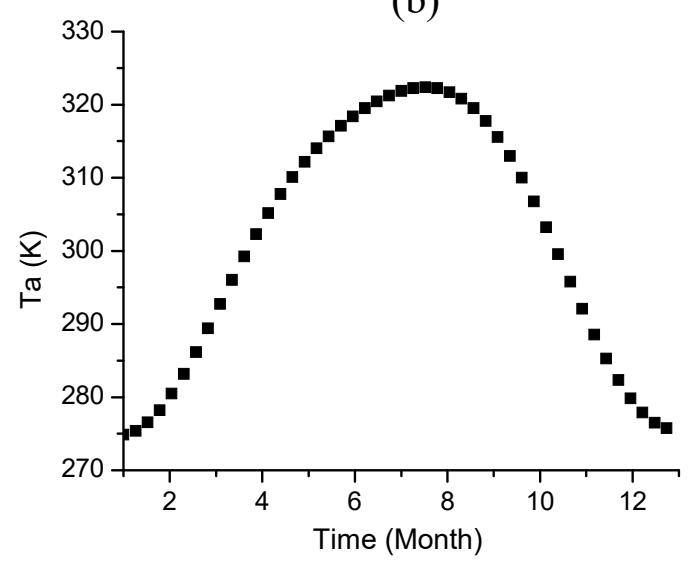

(c)

Figure A1. The case of obtaining the stable annual variation $\left(T_{\text {sta }}\right)$. (a) Land surface temperature at a fixed position; (b) annual variation in LST with annual periodicities in the range of 256-512 days; (c) stable annual variation field.

\section{Appendix D. Spatial Distribution of Land Surface Temperature and Its Components}

It can be seen from the procedure of LST processing that $T_{\text {local }}(t, x, y)$ is obtained from the LST and involves various temperature data, including $T_{a}(t, x, y), \Delta T(t, x, y), T_{\text {atmosphere }}(t, x, y), T_{\text {local }}(t, x, y)$ and $T_{\text {human }}(t, x, y)$. This article mainly analyzes $T_{\text {local }}(t, x, y)$ and does not involve other components. Here, we present a case for the spatial distribution of LST and its components.

To obtain more stable results, the data range selected in this paper is larger than the scope of the text. Here, we take the spatial results from the Aqua satellite as an example and give the spatial distributions of LST and its various temperature components (Figures A2-A7). As mentioned in Section 3.3, in order to avoid the border effect on the spatial decomposition, the spatial range is larger than those of Figure 2a, as shown in Figures A2-A7. 


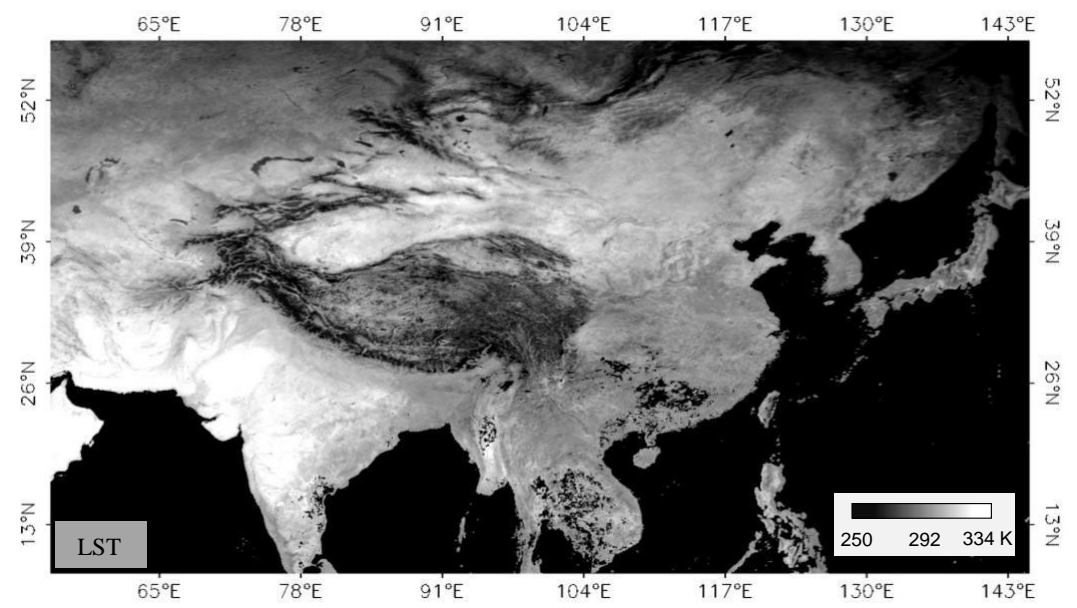

Figure A2. Land surface temperature at the time of the Nepal earthquake (Aqua) (23-30 April 2015).

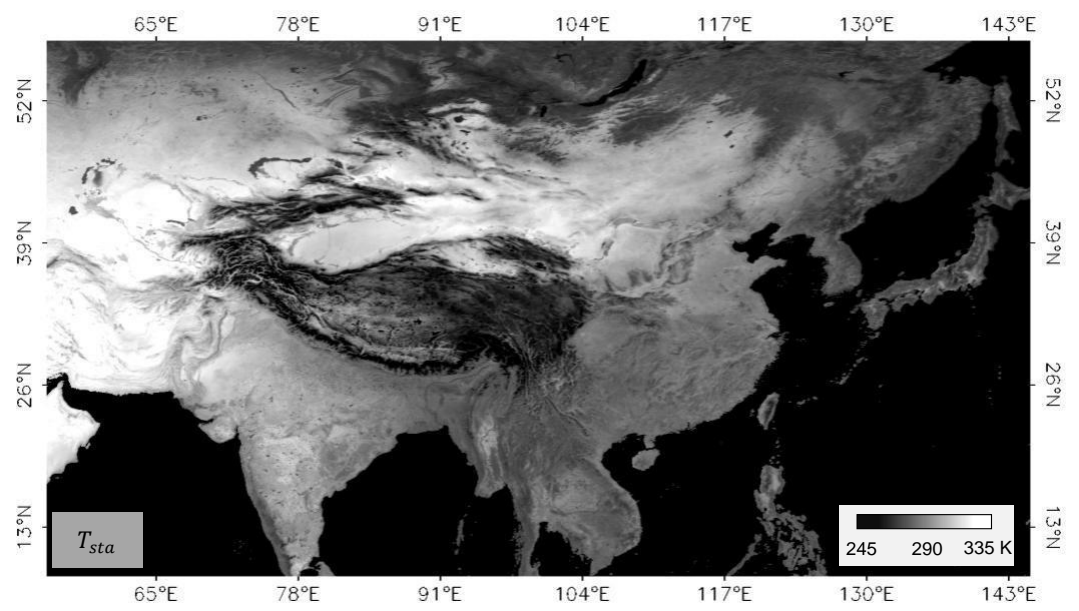

Figure A3. Spatial distribution of $T_{\text {sta }}$ (23-30 April 2015).

Figure A2 shows the LST at the occurrence of the Nepal earthquake. From the LST field, it is difficult to distinguish information related to earthquakes. Contrasting $T_{\text {sta }}(t, x, y)$ and $T(t, x, y)$ shows that the spatial distribution of the two is largely similar (Figures A2 and A3). The analysis of the time process illustrates that $T_{s t a}(t, x, y)$ is a stable annual cycle component that is independent of crustal activity and a stable static component that has the strongest influence on LST.

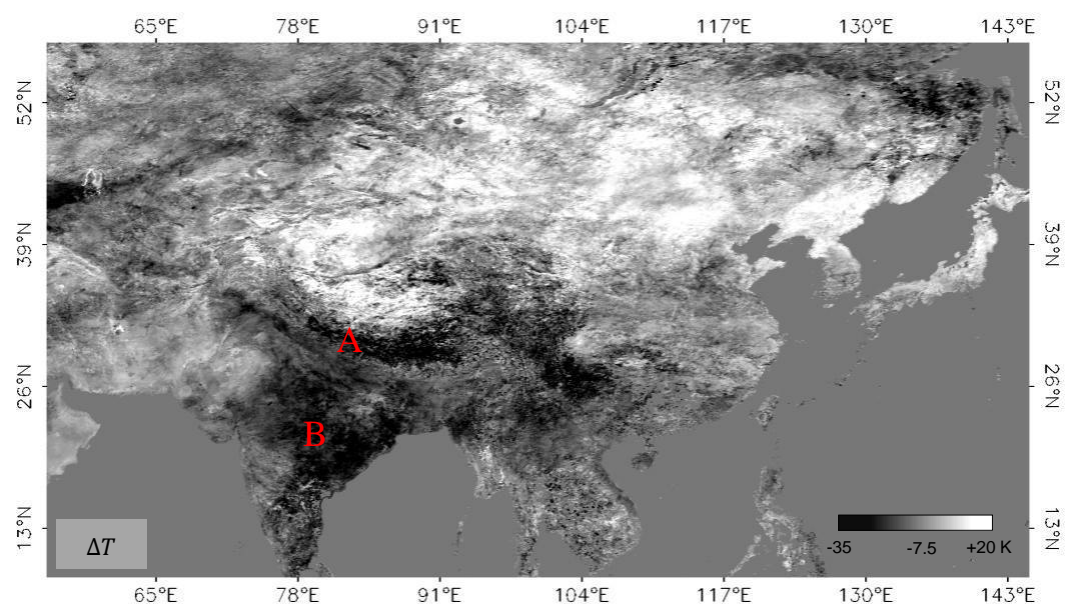

Figure A4. Spatial distribution of $\Delta T$ (23-30 April 2015). 
In $\Delta T(t, x, y)$, a temperature decrease (A) can be seen near the epicenter area (Figure A4). As shown in Figure A4, there are still other obvious cooling zones (B). Further, $\Delta T(t, x, y)$ is decomposed into $T_{\text {atmosphere }}(t, x, y), T_{\text {local }}(t, x, y)$ and $T_{\text {human }}(t, x, y)$, as shown in Figures A5-A7. The cooling zone B in Figure A4 is moved into $T_{\text {atmosphere }}(t, x, y)$ because it is more likely to be an atmospheric circulation effect. At the same time, in the spatial distribution of $T_{\text {local }}(t, x, y)$. the cooling phenomenon (A) near the epicenter is much clearer (Figure A7).

Note that the rectangular box in the spatial distribution of $T_{l o c a l}(t, x, y)$ in Figure A7 is the research range given in the main text (Figure 1a).

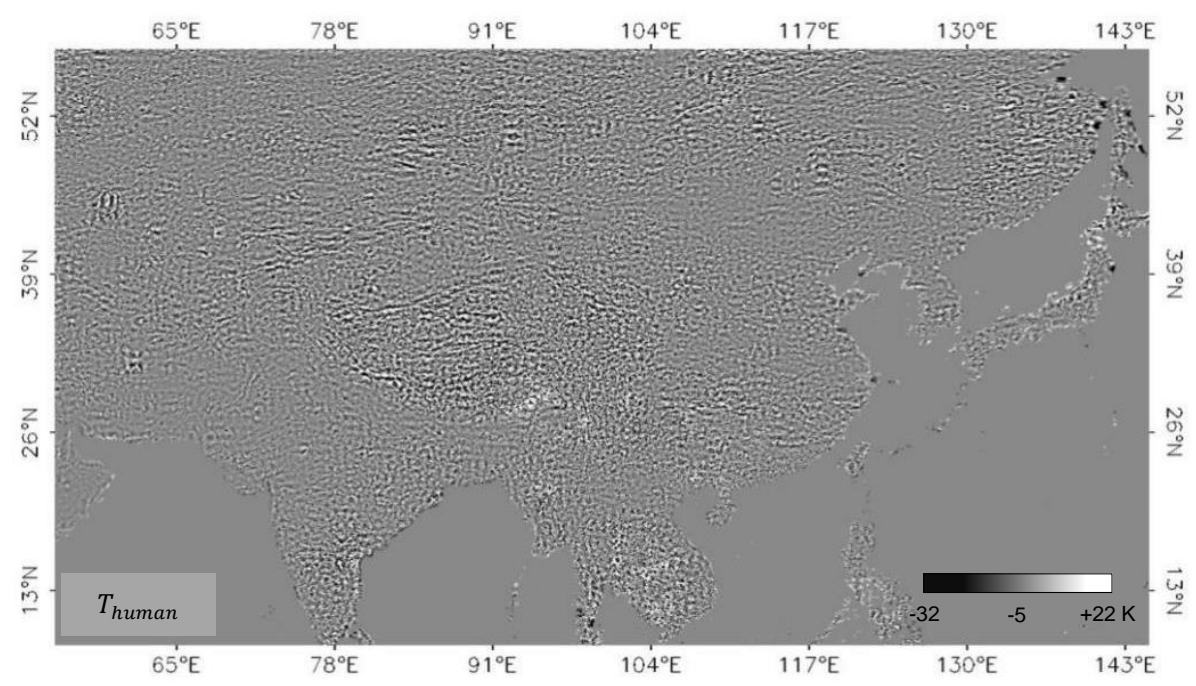

Figure A5. Spatial distribution of $T_{\text {human }}$. Spatial scales: less than $80 \mathrm{~km} \times 80 \mathrm{~km}$.

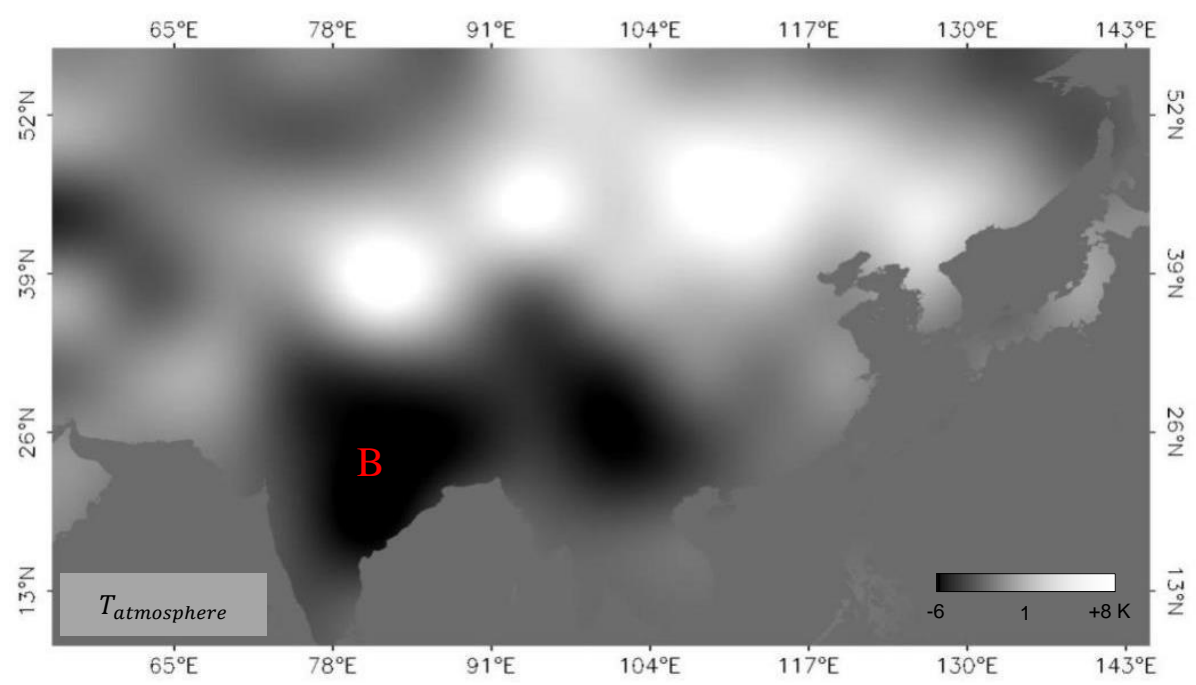

Figure A6. Spatial distribution of $T_{\text {atmosphere }}$. Spatial scales: greater than $600 \mathrm{~km} \times 600 \mathrm{~km}$. 


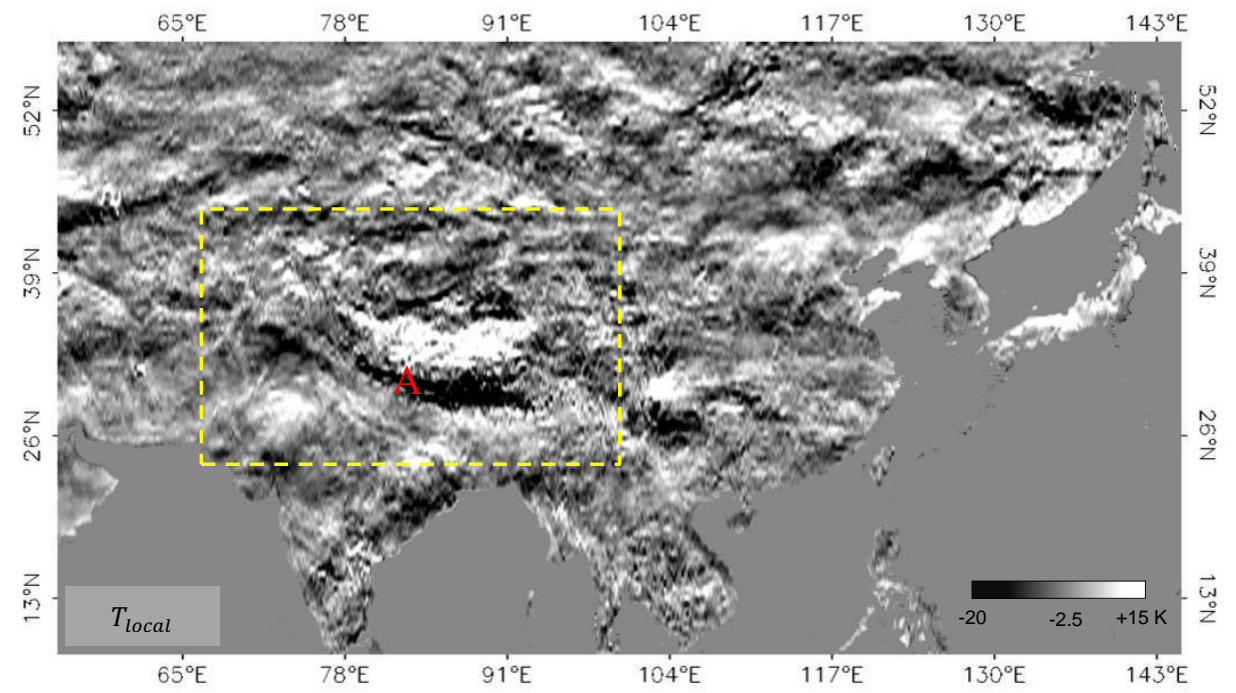

Figure A7. Spatial distribution of $T_{\text {local }}$. Spatial scales: greater than $80 \mathrm{~km} \times 80 \mathrm{~km}$ and less than $600 \mathrm{~km} \times 600 \mathrm{~km}$. Note: the rectangular box in $T_{\text {local }}$ is the research range given in the main text (namely, Figure 1a).

\section{References}

1. Geller, R.J. Earthquake prediction: A critical review. Geophys. J. Int. 1997, 131, 425-450. [CrossRef]

2. Geller, R.J.; Jackson, D.D.; Kagan, Y.Y.; Mulargia, F. Earthquakes Cannot Be Predicted. Science 1997, 275, 1616-1620. [CrossRef]

3. Ohnaka, M. The Physics of Rock Failure and Earthquakes; Cambridge University Press: New York, NY, USA, 2013.

4. Vallianatos, F.; Georgios, C. A Complexity View into the Physics of the Accelerating Seismic Release Hypothesis: Theoretical Principles. Entropy 2018, 20, 754. [CrossRef]

5. Song, D.; Xie, R.; Zang, L.; Yhi, J.; Qin, K.; Shan, X.; Cui, J.; Wang, B. A New Algorithm for the Characterization of Thermal Infrared Anomalies in Tectonic Activities. Remote Sens. 2018, 10, 1941. [CrossRef]

6. Jiao, Z.-H.; Zhao, J.; Shan, X. Pre-seismic anomalies from optical satellite observations: A review. Nat. Hazards Earth Syst. Sci. 2018, 18, 1013-1036. [CrossRef]

7. Varotsos, P.; Eftaxias, K.; Vallianatos, F.; Lazaridou, M. Basic principles for evaluating an earthquake prediction method. Transl. World Seismol. 1995, 23, 1295-1298. [CrossRef]

8. Qiang, Z.; Xu, X.; Dian, C. Satellite infrared thermos-anomaly: Earthquake imminent precursor. Chin. Sci. Bull. 1990, 35, 1324-1327.

9. Qiang, Z.J.; Kong, L.C.; Wang, Y.P. Earth gas emission, infrared thermo-anomaly and seismicity. Chin. Sci. Bull. 1992, 24, 2259-2262.

10. Tronin, A.A. Satellite thermal survey-A new tool for the studies of seismoactive regions. Int. J. Remote Sens. 1996, 17, 1439-1455. [CrossRef]

11. Tronin, A.A. Remote sensing and earthquakes: A review. Phys. Chem. Earth 2006, 31, 138-142. [CrossRef]

12. Tronin, A.A. Satellite Remote Sensing in Seismology: A Review. Remote Sens. 2010, 2, 124-150. [CrossRef]

13. Tronin, A.A.; Hayakawa, M.; Molchanov, O.A. Thermal IR satellite data application for earthquake research in Japan and China. J. Geodyn. 2002, 33, 519-534. [CrossRef]

14. Gorny, V.I.; Salman, A.G.; Tronin, A.A.; Shilin, B.B. Outgoing infrared radiation of the earth as an indicator of seismic activity. DoSSR 1988, 301, 67-69.

15. Ouzounov, D.; Freund, F.T. Mid-infrared emission prior to strong earthquakes analyzed by remote sensing data. Adv. Space Res. 2004, 33, 268-273. [CrossRef]

16. Ouzounov, D.; Liu, D.; Kang, C.; Guido, C.; Menas, K.; Patrick, T. Outgoing long wave radiation variability from IR satellite data prior to major earthquakes. Tectonophysics 2007, 431, 211-220. [CrossRef]

17. Tramutoli, V.; Cuomo, V.; Filizzola, C.; Pergola, N.; Pietrapertosa, C. Assessing the potential of thermal infrared satellite surveys for monitoring seismically active areas: The case of Kocaeli (Izmit) earthquake, August 17, 1999. Remote Sens. Environ. 2005, 96, 409-426. [CrossRef] 
18. Freund, F. Pre-earthquake signals-Part I: Deviatoric stresses turn rocks into a source of electric currents. Nat. Hazards Earth Syst. Sci. 2007, 7, 535-541. [CrossRef]

19. Freund, F. Pre-earthquake signals-Part II: Flow of battery currents in the Crust. Nat. Hazards Earth Syst. Sci. 2007, 7, 543-548. [CrossRef]

20. Freund, F. Pre-earthquake signals: Underlying physical processes. J. Asian Earth Sci. 2011, 41, $343-400$. [CrossRef]

21. Freund, F.; Takeuchi, A.; Lau, B.W.S.; Al-Manaseer, A.; Fu, C.C.; Bryant, N.A.; Ouzounov, D. Stimulated infrared emission from rocks: Assessing a stress indicator. eEarth 2007, 2, 1-10. [CrossRef]

22. Geng, N.G.; Cui, C.Y.; Deng, M.D. Remote sensing detection of rock fracturing experiment and the beginning of remote rock mechanics. Acta Seismol. Sin. 1992, 14, 645-652. (In Chinese)

23. Saraf, A.K.; Rawat, V.; Choudhury, S.; Dasgupta, S.; Das, J. Advances in understanding of the mechanism for generation of earthquake thermal precursors detected by satellites. Int. J. Appl. Earth Obs. Geoinf. 2009, 11, 373-379. [CrossRef]

24. Eleftheriou, C.; Filizzola, N.; Genzano, T.; Lacava, M.; Lisi, R.; Paciello, N.; Pergola, F.; Vallianatos, V. Tramutoli. Long-Term RST Analysis of Anomalous TIR Sequences in Relation with Earthquakes Occurred in Greece in the Period 2004-2013. Pure Appl. Geophys. 2016, 173, 285-303. [CrossRef]

25. Bhardwaj, A.; Singh, S.; Sam, L.; Bhardwaj, A.; Martín-Torres, F.J.; Singh, A.; Kumar, R. MODIS-based estimates of strong snow surface temperature anomaly related to high altitude earthquakes of 2015. Remote Sens. Environ. 2017, 188, 1-8. [CrossRef]

26. Blackett, M.; Wooster, M.; Malamud, B. Exploring land surface temperature earthquake precursors: A focus on the Gujarat (India) earthquake of 2001. Geophys. Res. Lett. 2011, 38. [CrossRef]

27. Chen, S.Y.; Ma, J.; Liu, P.X.; Liu, L.Q.; Ren, Y.Q. Exploring the current tectonic activity with satellite remote sensing thermal information: A case of the Wenchuan earthquake. Seismol. Geol. 2014, 36, 775-793.

28. Liu, J.; Chen, J.I.; Zhang, J.Y.; Zhang, P.Z.; Wei, W. Tectonic setting and general features of coseismic rupture of the 25 April, 2015 Mw 7.8 Gorkha Nepal earthquake (in Chinese). Chin. Sci. Bull. 2015, 60, 2640-2655. [CrossRef]

29. Yang, X.-P.; Wu, G.; Chen, L.-C.; Li, C.-Y.; Chen, X.-L. The seismogenic structure of the April 25, 2015 MW7.8 Nepal earthquake in the southern margin of Qinghai-Tibetan Plateau. Chin. J. Geophys. 2016, 59, 2528-2538.

30. Nandita, D.G. Atmospheric changes observed during April 2015 Nepal earthquake. J. Atmos. Sol. Terr. Physics. 2016, 140, 16-22.

31. Chakraborty, S.; Sasmal, S.; Chakrabarti, S.K.; Bhattacharya, A. Observational signatures of unusual outgoing longwave radiation (OLR) and atmospheric gravity waves (AGW) as precursory effects of May 2015 Nepal earthquakes. J. Geodyn. 2018, 113, 43-51. [CrossRef]

32. Molnar, P.; Tapponnier, P. Cenozoic tectonics of Asia: Effects of a continental collision. Science 1975, 189, 419-426. [CrossRef] [PubMed]

33. Nakata, T. Active faults of the Himalaya of India and Nepal. GSA Spec. Pap. 1989, 232, 243-261.

34. Ni, J.; Barazangi, M. Seismotectonics of the Himalayan Collision Zone: Geometry of the underthrusting Indian Plate beneath the Himalaya. J. Geophys. Res. Solid Earth 1984, 89, 1147. [CrossRef]

35. Brown, L.; Zhao, W.; Nelson, K.; Hauck, M.; Alsdorf, D.; Ross, A.; Cogan, M.; Clark, M.; Liu, X.; Che, J. Bright spots, Structure, and Magmatism in Southern Tibet from INDEPTH Seismic Reflection Profiling. Science 1996, 274, 1688-1690. [CrossRef]

36. Zhang, X.; Xu, L.-S. Inversion of the apparent source time functions for the rupture process of the Nepal Ms8.1 earthquake. Chin. J. Geophys. 2015, 58, 1881-1890. (In Chinese)

37. Lay, T.; Ye, L.; Koper, K.D.; Kanamori, H. Assessment of teleseismically-determined source parameters for the April 25, 2015 MW 7.9 Gorkha, Nepal earthquake and the May 12, 2015 MW 7.2 aftershock. Tectonophysics 2017, 714, 4-20. [CrossRef]

38. Denolle, M.A.; Fan, W.; Shearer, P.M. Dynamics of the 2015 M7.8 Nepal earthquake. Geophys. Res. Lett. 2015, 42, 7467-7475. [CrossRef]

39. Wan, Z.; Li, Z.L. A physics-based algorithm for retrieving land surface emissivity and temperature from EOS/MODIS data. IEEE Trans. Geosci. Remote Sens. 1997, 35, 980-996.

40. Wan, Z.M. New refinements and validation of the MODIS Land Surface Temperature/Emissivity products. Remote Sens. Environ. 2008, 112, 59-74. [CrossRef] 
41. Wan, Z.M.; Zhang, Y.L.; Zhang, Q.H.; Li, Z. Validation of the land surface temperature products retrieved from Terra Moderate Resolution Imaging Spectroradiometer data. Remote Sens. Environ. 2002, 83, 163-180. [CrossRef]

42. Li, J.; Tang, Y. Application of Wavelet Analyses; Chongqing University Press: Chongqing, China, 1999. (In Chinese)

43. Mallat, S. A theory of multi-resolution signal decomposition: The wavelet representation. IEEE Trans. PAMI 1989, 11, 674-693. [CrossRef]

44. Wu, L.; Cui, C.; Geng, N.; Wang, J. Remote sensing rock mechanics (RSRM) and associated experimental studies. Int. J. Rock Mech. Min. Sci. 2000, 37, 879-888. [CrossRef]

45. Yin, J.Y.; Fang, Z.F.; Qian, J.D.; Deng, M.D.; Geng, N.G.; Hao, J.S.; Wang, Z.; Ji, Q.Q. Research on the application of infrared remote sensing in earthquake prediction and its physical mechanism. Earthq. Res. China 2000, 16, 140-148.

46. Pulinets, S.; Ouzounov, D.; Karelin, A.; Boyarchuk, K.; Pokhmelnykh, L. The physical nature of thermal anomalies observed before strong earthquakes. Phys. Chem. Earth Part A/B/C 2006, 31, 143-153. [CrossRef]

47. Ren, Y.; Chen, S.; Ma, J. Variation of land surface temperature in Yilan-Yitong fault zone of northeastern China. Acta Seismol. Sin. 2012, 34, 698-705.

48. Chen, S.; Liu, P.; Guo, Y.; Liu, L.; Ma, J. Co-Seismic Response of Bedrock Temperature to the Ms6.3 Kangding Earthquake on 22 November 2014 in Sichuan, China. Pure Appl. Geophys. 2019, 176, 97-117. [CrossRef]

49. Chen, S.Y.; Liu, L.; Liu, P.; Ma, J.; Ghen, G. Theoretical and experimental study on relationship between stress-strain and temperature variation. Sci. China Ser. D Earth Sci. 2009, 52, 1825-1834. [CrossRef]

50. Liu, P.; Chen, S.; Liu, L.; Chen, G.; Ma, J. An experiment on the infrared radiation of surficial rocks during deformation. Seismol. Geol. 2004, 26, 502-511, (In Chinese with English abstract).

51. Yang, X.; Lin, W.; Tadai, O.; Zeng, X.; Yu, C.; Yeh, E.-C.; Li, H.; Wang, H. Experimental and numerical investigation of the temperature response to stress changes of rocks. J. Geophys. Res. Solid Earth 2017, 122, 5101-5117. [CrossRef]

52. Chui, C.K. An Introduction to Wavelets; Academic Press: San Diego, CA, USA, 1992.

53. Daubechies, I. Ten Lectures on Wavelets; SIAM Press: Philadelphia, PA, USA, 1992.

54. Boggess, A.; Narcowich, F.J. A First Course in Wavelets with Fourier Analysis; John Wiley \& Sons, Inc.: Hoboken, NJ, USA, 2009.

55. Chen, S.-Y.; Ma, J.; Liu, P.-X.; Liu, L.-Q. A study on the normal annual variation field of land surface temperature in China. Chin. J. Geophys. 2009, 52, 962-971. [CrossRef] 